\title{
Ducks on the torus: existence and uniqueness
}

\author{
Ilya V. Schurov*
}

\begin{abstract}
We show that there exist generic slow-fast systems with only one (time-scaling) parameter on the two-torus, which have canard cycles for arbitrary small values of this parameter. This is in drastic contrast with the planar case, where canards usually occur in two-parametric families. Here we treat systems with a convex slow curve. In this case there is a set of parameter values accumulating to zero for which the system has exactly one attracting and one repelling canard cycle. The basin of the attracting cycle is almost the whole torus.
\end{abstract}

UDC 517.925.42+517.938.

AMS MSC 2010: 70K70, 37G15.

Keywords: slow-fast systems, canards, limit cycles, Poincaré map, distortion lemma.

\section{Introduction}

Consider a generic slow-fast system on the plane:

$$
\left\{\begin{array}{l}
\dot{x}=f(x, y, \varepsilon) \\
\dot{y}=\varepsilon g(x, y, \varepsilon)
\end{array} \quad(x, y) \in \mathbb{R}^{2}, \quad \varepsilon \in(\mathbb{R}, 0) .\right.
$$

There is a rather simple description of its behavior for small $\varepsilon$. It consists of interchanging phases of slow motion along stable parts of the slow curve

${ }^{*}$ Moscow State University, Mechanics and mathematics department, Leninskie gory, Moscow, 119991. E-mail: ilya@schurov.com. The author is supported in part by RFBR and CNRS (project 05-01-02801-CNRS-L-a) and RFBR project 07-01-00017-a 
$M:=\{(x, y) \mid f(x, y, 0)=0\}$ and fast jumps along straight lines $y=$ const. Given additional parameters, which depend on $\varepsilon$, one can observe more complicated behavior: appearance of duck (or canard) solutions (particularly limit cycles), i.e. solutions, whose phase curves contain an arc of length bounded away from 0 uniformly in $\varepsilon$, that keeps close to the unstable part of the slow curve (see [Di] and [DR]).

In GI], Yu. S. Ilyashenko and J. Guckenheimer discovered a new kind of behavior of slow-fast systems on the two-torus. It was shown that for some particluar family with no auxiliary parameters there exists a sequence of intervals accumulating at 0 , such that for any $\varepsilon$ from these intervals, the system has exactly two limit cycles, both of which are canards, where one is stable and the other unstable.

Yu. S. Ilyashenko and J. Guckenheimer conjectured that there exists an open domain in the space of slow-fast systems on the two-torus with the same property. This work is devoted to the proof of this conjecture: we generalize the result of [GI] for generic slow-fast systems with convex slow curve. The work is based on the ideas of [GI] and has similar structure: Section 2 states the Main Theorem and outlines its proof, consisting of a sequence of auxiliary lemmas. In Section 3 we state necessary theorems about normal forms of slow-fast systems. The lemmas are proved in Section 4, and some auxiliary propositions are proved in the Appendix (Section 5).

The author would like to express his sincere appreciation to $\mathrm{Yu}$. S. Ilyashenko for the statement of the problem and his assistance with the work, to A. Fishkin for assistance with the work, to V. Kleptsyn for fruitful discussions, valuable comments on the text of the work and idea of using Distortion Lemma, to G. Kolutsky for valuable comments on the text of the work. The author also grateful to the anonymous referee for valuable comments.

\section{Slow-fast systems on the two-torus and Poincaré map}

\subsection{Preliminary statement of the main result}

Consider a slow-fast system on the two-torus:

$$
\left\{\begin{array}{l}
\dot{x}=f(x, y, \varepsilon) \\
\dot{y}=\varepsilon g(x, y, \varepsilon)
\end{array} \quad(x, y) \in \mathbb{T}^{2} \cong \mathbb{R}^{2} /\left(2 \pi \mathbb{Z}^{2}\right), \varepsilon \in(\mathbb{R}, 0),\right.
$$

where functions $f$ and $g$ are assumed to be smooth enough. 
The following theorem is a corollary of the main result (Theorem 2.5 below):

Theorem 2.1. There exists an open set in the space of slow-fast systems on the two-torus with the following property. For every system from this set there exists a sequence of intervals accumulating at zero, such that for every $\varepsilon$ that belongs to these intervals the system has an attracting canard cycle. The basin of this cycle is the whole torus excluding exactly one unstable cycle.

A rigorous definition of the term "canard solution" as well as conditions that define the open set mentioned in the theorem above are given in the next section. The main result (Theorem 2.5) is a stronger version of Theorem 2.1.

\subsection{Full statement of the main result}

For the slow-fast system (2.1) denote its slow curve by $M$ :

$$
M:=\{(x, y) \mid f(x, y, 0)=0\}
$$

Impose the following conditions of local genericity on system (2.1):

1. The speed of the slow motion is bounded away from zero: $g>0$.

2. $M$ is a smooth curve.

3. The lift of the curve $M$ to the covering coordinate plane is contained in the interior of the fundamental square $\{|x|<\pi,|y|<\pi\}$ and is convex. This, in particular, implies that there are two jump points (straight and inverse jumps), which are the far right and the far left points of $M$. (See Fig. 2.1.) We denote them $G^{-}$and $G^{+}$respectively.

4. The following nondegenericity assumption holds in every point $(x, y) \in$ $M \backslash\left\{G^{+}, G^{-}\right\}:$

$$
\frac{\partial f(x, y, 0)}{\partial x} \neq 0
$$

5. The following nondegenericity assumptions hold in the jump points:

$$
\left.\frac{\partial^{2} f(x, y, 0)}{\partial x^{2}}\right|_{G^{ \pm}} \neq 0,\left.\quad \frac{\partial f(x, y, 0)}{\partial y}\right|_{G^{ \pm}} \neq 0
$$

Conditions 15 define an open set in the space of slow-fast systems on the two-torus. 


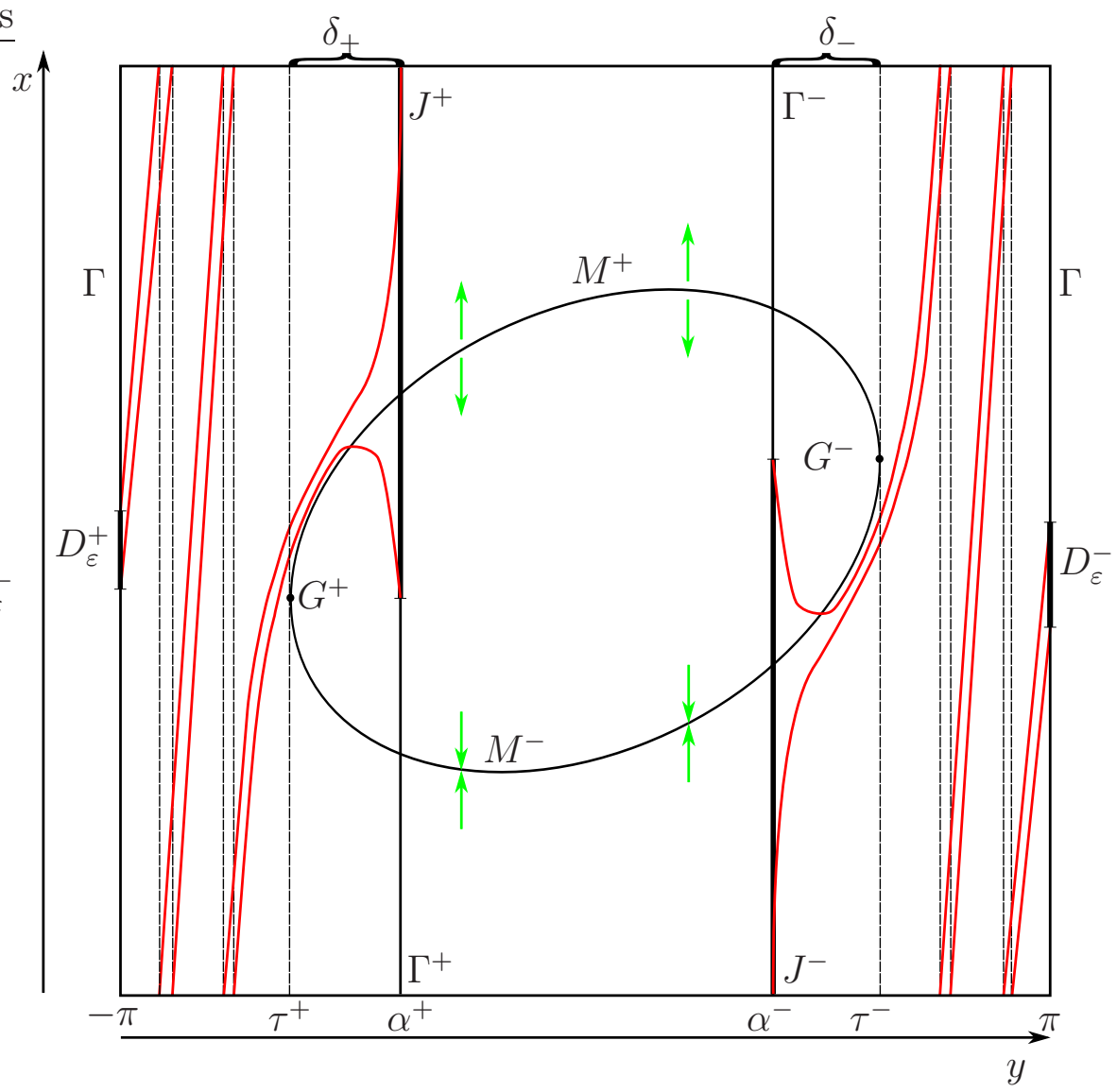

Figure 2.1: Slow-fast system on the two-torus. $x$-axis is directed upward, red lines are trajectories of the system.

Remark 2.2. Without loss of generality (by altering the direction of the $x$-axis if necessary) we can assume that:

$$
\begin{array}{lll}
\left.\frac{\partial f(x, y, 0)}{\partial y}\right|_{G^{+}}<0, & \left.\frac{\partial^{2} f(x, y, 0)}{\partial x^{2}}\right|_{G^{+}}>0, \\
\left.\frac{\partial f(x, y, 0)}{\partial y}\right|_{G^{-}}>0, & \left.\frac{\partial^{2} f(x, y, 0)}{\partial x^{2}}\right|_{G^{-}}>0 .
\end{array}
$$

Let $M^{ \pm}$be the stable (-) and unstable $(+)$parts of the slow curve. Let us fix a vertical interval $J^{+}$that crosses the unstable part of the slow curve $M^{+}$near the jump point $G^{+}$and does not intersect $M^{-}$(the exact position of this interval will be specified later).

Defenition 2.3. Any solution that crosses $J^{+}$is a canard solution. 
In order to construct canard cycles, we will study the Poincaré map $P_{\varepsilon}$ from the global cross-section $\Gamma=\{y=-\pi\}$ to itself along the trajectories of system (2.1). As the function $g$ is bounded away from zero, this map is a welldefined diffeomorphism of a circle to itself. Note that periodic trajectories of system (2.1) correspond to periodic (in particular, fixed) points of this map; thus, it is natural to consider the rotation number of $P_{\varepsilon}$. Let us lift $P_{\varepsilon}$ to universal cover and denote the rotation number of lifted map by $\rho(\varepsilon) \in \mathbb{R}$.

Remark 2.4. Hereafter by "the assertion holds for small $\varepsilon$ " we shall mean that the following condition is satisfied: there exists an $\varepsilon_{0}>0$ such that the assertion holds for every $\varepsilon \in\left(0, \varepsilon_{0}\right]$. We can choose a universal $\varepsilon_{0}$ for all such assertions in the paper.

Theorem 2.5 (Main result). For any system (2.1) that satisfies conditions 1 15, there exists a sequence of non-intersecting intervals $\left\{R_{n}\right\}, R_{n}=\left[\alpha_{n}, \beta_{n}\right]$ and two sequences of non-intersecting intervals $C_{n}^{ \pm} \subset R_{n}$ with the following properties:

1. $\left|R_{n}\right|=O\left(e^{-C n}\right)$ for some $C>0$.

2. $\alpha_{n}=O(1 / n)$

3. For every $\varepsilon$ sufficiently small, not belonging to the $R_{n}$ 's, the rotation number $\rho(\varepsilon)$ is an integer. For such $\varepsilon$ there are exactly two periodic trajectories, both of which are hyperbolic, where one is stable and the other unstable. The unstable one is a canard.

4. For every sufficiently small $\varepsilon \in C_{n}^{ \pm}$, system (2.1) has exactly two periodic trajectories, both of which are hyperbolic (where one stable and one unstable), and both are canards.

Remark 2.6. The condition of convexity of $M$ above can be weakened to that of the existence of exactly two points of $M$ with a vertical tangent line. Indeed, using a smooth coordinate change that preserves vertical circles, any such curve can be made convex. On the other hand, such a change will not affect the conditions of local genericity.

As it was mentioned above, the main tool of our study will be the Poincaré map $P_{\varepsilon}$. In Section 2.3 three Lemmas (2.8, 2.10 and 2.12) are stated. They describe the behavior of the Poincaré map as $\varepsilon \rightarrow 0^{+}$. In section 2.4, the Main Theorem 2.5 is proved modulo these Lemmas. 


\subsection{Poincaré map}

Remark 2.7. Note that the function $g$ is bounded away from zero, so we can divide the system (2.1) by it, thus re-scaling the time: this does not change the desired properties of its solutions, and the system with new function $f$ still belongs to the same open set. Thus without loss of generality we can assume $g=1$ in (2.1) and consider the system:

$$
\left\{\begin{array}{l}
\dot{x}=f(x, y, \varepsilon) \\
\dot{y}=\varepsilon
\end{array} \quad(x, y) \in \mathbb{T}, \varepsilon \in(\mathbb{R}, 0)\right.
$$

Denote the graph of the Poincaré map $P_{\varepsilon}$ by $\gamma_{\varepsilon} \subset S^{1} \times S^{1}$. The following lemma shows that this graph looks more and more like the union of a horizontal and a vertical circle as $\varepsilon$ tends to $0^{+}$. In other words, the derivative of the Poincaré map is (exponentially) small on the whole $\Gamma$ with the exception of an exponentially small interval.

Lemma 2.8 (Shape Lemma). There exist constants $c_{1,2}^{ \pm}>0$ such that for any sufficiently small $\varepsilon>0$ one can find two intervals $D_{\varepsilon}^{+}$and $D_{\varepsilon}^{-}$in the preimage and image of $P_{\varepsilon}$ resp. with the following properties:

1. $\left|D_{\varepsilon}^{ \pm}\right|=O\left(e^{-c_{1}^{ \pm} / \varepsilon}\right)$

2. $\mid P_{\varepsilon}^{\prime} \|_{S^{1} \backslash D_{\varepsilon}^{+}}=O\left(e^{-c_{2}^{+} / \varepsilon}\right)$

3. $\left.\left|\left(P_{\varepsilon}^{-1}\right)^{\prime}\right|\right|_{S^{1} \backslash D_{\varepsilon}^{-}}=O\left(e^{-c_{2}^{-} / \varepsilon}\right)$

4. The graph $\gamma_{\varepsilon}$ lies in the union of two orthogonal rings: $\Pi_{\varepsilon}^{+}:=D_{\varepsilon}^{+} \times S^{1}$ and $\Pi_{\varepsilon}^{-}:=S^{1} \times D_{\varepsilon}^{-}$.

Lemma 2.8 is proved in Section 4.1. The next lemma formalizes the following observation: the lift of $\gamma_{\varepsilon}$ to the fundamental domain $\{|x|<\pi,|y|<$ $\pi\}$ moves monotonically to the upper left as $\varepsilon \rightarrow 0^{+}$. To formalize this, we need to introduce some additional notation.

Consider arbitrary points $a$ and $b$ on the oriented circle $S^{1}$. They split the circle into two arcs. Denote the arc from point $a$ to point $b$ (in the sense of the orientation of the circle) by $[a, b\rangle$. The orientation of this arc is induced by the orientation of the circle. Also denote the same arc with the inversed orientation by $\langle a, b]$. (See fig. [2.2.)

Denote the Poincaré map along the phase curves of the main system (2.1) from the cross-section $y=a$ to the cross-section $y=b$ in the forward time (i.e. along the $\operatorname{arc}[a, b\rangle)$ by $P_{\varepsilon}^{[a, b\rangle}$. Also, let $P_{\varepsilon}^{\langle a, b]}=\left(P_{\varepsilon}^{[a, b\rangle}\right)^{-1}$ : this is the 


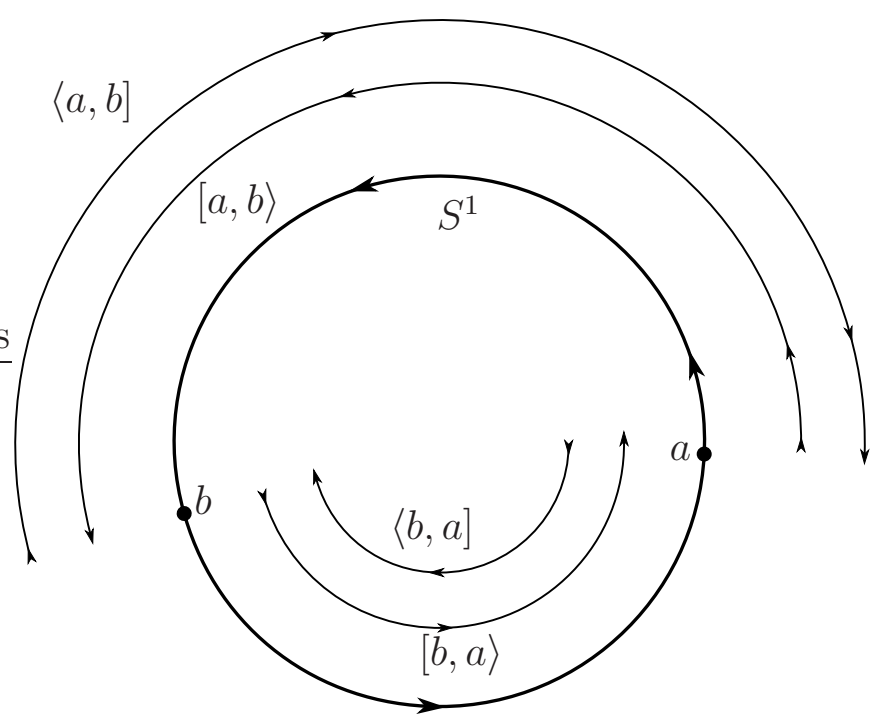

Figure 2.2: Orientation of the arcs

Poincaré map from the cross-section $y=b$ to the cross-section $y=a$ in the reverse time. This fact is stressed by the notation: the direction of the angle bracket shows the time direction.

Hereafter any formula containing a \pm or $\mp$ sign replaces two formulae: one with all the upper sign and another with all the lower sign.

Let the jump points $G^{ \pm}$have coordinates $\left(\sigma^{ \pm}, \tau^{ \pm}\right)$resp., (the slow curve $M$ lies inside the strip $\left\{\tau^{+} \leq y \leq \tau^{-}\right\}$due to its convexity, see Fig. 2.1). For some fixed small positive $\delta_{+}$and $\delta_{-}$we define the following objects:

1. Transversal cross-sections $\Gamma^{ \pm}$, intersecting the slow curve $M$ near the jump points:

$$
\Gamma^{ \pm}:=S^{1} \times\left\{\alpha^{ \pm}\right\}
$$

where $\alpha^{ \pm}=\tau^{ \pm} \pm \delta_{ \pm}$. (See Fig. 2.1.)

2. Segments $J^{+}$and $J^{-}$of the cross-sections $\Gamma^{ \pm}$, which intersect unstable and stable parts of the slow curve resp.:

$$
\begin{gathered}
J^{+}:=\left\{\left(x, \alpha^{+}\right) \in \Gamma^{+} \mid x \in\left[\sigma^{+}, \pi\right]\right\}, \\
J^{-}:=\left\{\left(x, \alpha^{-}\right) \in \Gamma^{-} \mid x \in\left[-\pi, \sigma^{-}\right]\right\} .
\end{gathered}
$$

3. Segments $D_{\varepsilon}^{ \pm}$(whose existence is provided by the Shape Lemma 2.8):

$$
D_{\varepsilon}^{+}:=P_{\varepsilon}^{\left\langle-\pi, \alpha^{+}\right]}\left(J^{+}\right), \quad D_{\varepsilon}^{-}:=P_{\varepsilon}^{\left[\alpha^{-}, \pi\right\rangle}\left(J^{-}\right), \quad D_{\varepsilon}^{ \pm}=\left[p_{\varepsilon}^{ \pm}, q_{\varepsilon}^{ \pm}\right\rangle,
$$


The choice of segment $J^{+}$here formally completes Definition 2.3 that used this interval. Note that all trajectories that intersect segment $D_{\varepsilon}^{+}$also intersect $J^{+}$and thus are canards.

Let $B_{\varepsilon}^{+}$and $B_{\varepsilon}^{-}$be the points on the graph $\gamma_{\varepsilon}$, where the slope of $P_{\varepsilon}$ is equal to 1 . As it will be shown below (see Lemma 2.12) there are exactly two such points. From the Lemma 2.8 it follows that the points $B_{\varepsilon}^{ \pm}$lie in the rectangle $K_{\varepsilon}:=D_{\varepsilon}^{+} \times D_{\varepsilon}^{-}$, because outside of this rectangle the derivative of $P_{\varepsilon}$ is either very small or very big. For the sake of definiteness, we will assume that $\left[x\left(B_{\varepsilon}^{-}\right), x\left(B_{\varepsilon}^{+}\right)\right\rangle \subset D_{\varepsilon}^{+}$: let us denote this as $x\left(B_{\varepsilon}^{-}\right)<x\left(B_{\varepsilon}^{+}\right)$.

Remark 2.9. The map $P_{\varepsilon}$ preserves orientation (i.e. monotonic) and is bijective, so $\left[y\left(B_{\varepsilon}^{+}\right), y\left(B_{\varepsilon}^{-}\right\rangle\right) \subset D_{\varepsilon}^{-}$(i.e. $\left.y\left(B_{\varepsilon}^{-}\right)>y\left(B_{\varepsilon}^{+}\right)\right)$.

Let us denote by $A_{\varepsilon}^{+}$and $A_{\varepsilon}^{-}$the points of the graph $\gamma_{\varepsilon}$ which lie above the ends of the segment $D_{\varepsilon}^{+}$(see figure 2.3):

$$
A_{\varepsilon}^{-}:=\left(p_{\varepsilon}^{+}, P_{\varepsilon}\left(p_{\varepsilon}^{+}\right)\right), \quad A_{\varepsilon}^{+}:=\left(q_{\varepsilon}^{+}, P_{\varepsilon}\left(q_{\varepsilon}^{+}\right)\right),
$$

Denote also the top left and bottom right (in the sense of orientations of coordinate circles) corners of the rectangle $K_{\varepsilon}$ by $E_{\varepsilon}^{-}$and $E_{\varepsilon}^{+}$resp.:

$$
E_{\varepsilon}^{-}:=\left(p_{\varepsilon}^{+}, q_{\varepsilon}^{-}\right), \quad E_{\varepsilon}^{+}:=\left(q_{\varepsilon}^{+}, p_{\varepsilon}^{-}\right)
$$

Let $C_{\varepsilon}$ stand for either of the points $B_{\varepsilon}^{ \pm}, A_{\varepsilon}^{ \pm}$or $E_{\varepsilon}^{ \pm}$and let $\bar{C}_{\varepsilon}$ be its lift to the universal cover depending continuosly on $\varepsilon$.

Lemma 2.10 (Monotonicity lemma). The following assertions hold:

1. $\frac{d}{d \varepsilon}(x-y)\left(\bar{C}_{\varepsilon}\right) \rightarrow+\infty$ as $\varepsilon \rightarrow 0^{+}$, for any choice $\bar{C}_{\varepsilon}=\bar{A}_{\varepsilon}^{ \pm} ; \bar{E}_{\varepsilon}^{ \pm}$

2. The equation $(y-x)\left(\bar{E}_{\varepsilon}^{-}\right)=2 \pi n$ has solution $\varepsilon=\varepsilon_{n}$ for any $n$, and $\varepsilon_{n}=O(1 / n)$.

Remark 2.11. The second assertion of the Lemma implies that for $\varepsilon=\varepsilon_{n}$ the diagonal

$$
\Delta:=\{y=x \quad(\bmod 2 \pi \mathbb{Z})\}
$$

crosses top left corner of the rectangle $K_{\varepsilon}$.

Lemma 2.10 is proved in Section 4.3 .

The following lemma describes the graph of the Poincaré map near the points $B_{\varepsilon}^{ \pm}$. Denote by $U$ the set of points on $\Gamma$ where the derivative of the Poincaré map is close to 1 :

$$
U:=\left\{x \in S^{1} \mid P_{\varepsilon}^{\prime}(x) \in[1 / 2,2]\right\}
$$


Lemma 2.12 (Convexity Lemma). The set $U$ consists of two arcs contained in $D_{\varepsilon}^{+}$. On one of them the derivative $P_{\varepsilon}^{\prime}$ increases and on the other it decreases.

In particular, it follows that there exist exactly two solutions of the equation $P_{\varepsilon}^{\prime}(x)=1$. Lemma 2.12 is proved in the Section 4.2.

Now we can prove Main Theorem 2.5 modulo these lemmas.

\subsection{Existence of canard solutions}

In this section we deduce Main Theorem 2.5 from Lemmas 2.8, 2.10 and 2.12.

\subsubsection{Heuristic ideas}

We notice that the points of intersection of the graph $\gamma_{\varepsilon}$ and the diagonal $\Delta:=\{y=x(\bmod 2 \pi \mathbb{Z})\}$ correspond to the fixed points of the Poincaré map and therefore to the closed solutions of the system. The stability of the corresponding cycle depends on the derivative of the Poincaré map at the fixed point: if the derivative is greater than 1, then the cycle is unstable, if it is less then 1, then the cycle is stable. We are particulary interested in the fixed points that belong to the segment $D_{\varepsilon}^{+}$because they correspond to the closed canard solutions.

Lemmas 2.8 and 2.10 describe behavior of the system as $\varepsilon \rightarrow 0^{+}$. The graph $\gamma_{\varepsilon}$ lies inside the union of horizontal and vertical rings, which are exponentially thin when $\varepsilon \rightarrow 0^{+}$. The graph moves from the lower right corner to the upper left. Theoretically, the following cases for relative positions of the diagonal $\Delta$ and the graph $\gamma_{\varepsilon}$ are possible (Fig. 2.3):

1. The diagonal $\Delta$ does not intersect the rectangle $K_{\varepsilon}$. In this case it intersects $\gamma_{\varepsilon}$ in two points, one of them belongs to the ring $\Pi_{\varepsilon}^{+}$, and corresponds to an unstable fixed point, and the other belongs to the ring $\Pi_{\varepsilon}^{-}$and corresponds to the stable fixed point from $\Gamma \backslash D_{\varepsilon}^{+}$.

2. The diagonal $\Delta$ intersects the rectange $K_{\varepsilon}$, but the stable fixed point is located outside $D_{\varepsilon}^{+}$like in the previous case. (Note that the position of the unstable fixed point is not significant for our analysis.)

3. The diagonal $\Delta$ intersects $\gamma_{\varepsilon}$ in two points, and the point of interstection which corresponds to the stable fixed point belongs to the rectangle $K_{\varepsilon}$. In this case this point corresponds to the stable canard cycle, which provides its existence in Main Theorem, 2.5. Uniqueness of the cycle follows from Convexity Lemma 2.12.

4. The diagonal $\Delta$ touches $\gamma_{\varepsilon}$ in one of the points $B_{\varepsilon}^{ \pm}$.

5. The diagonal $\Delta$ does not intersect $\gamma_{\varepsilon}$. 
From Lemma 2.10 and the fact that the graph $\gamma_{\varepsilon}$ depends on $\varepsilon$ continuosly it follows that when $\varepsilon \rightarrow 0^{+}$, the described cases occur consecutively and cyclically, in the following order: $1,2,3,4,5,4,3,2,1, \ldots$ Values of $\varepsilon$ that correspond to case 3 form intervals $C_{n}^{ \pm}$mentioned in Main Theorem 2.5.

Later we will prove these propositions strictly.

\subsubsection{Segments on the line of $\varepsilon$}

Proof of Main Theorem 2.5. Define the segments $R_{n}$ in the following way:

$$
R_{n}:=\left\{\varepsilon \in\left(\mathbb{R}_{+}, 0\right) \mid(y-x)\left(\bar{E}_{\varepsilon}^{+}\right) \leq 2 \pi n \leq(y-x)\left(\bar{E}_{\varepsilon}^{-}\right)\right\}=\left[\alpha_{n}, \beta_{n}\right]
$$

In other words, $\varepsilon \in R_{n}$ when the diagonal $\Delta$ crosses the rectangle $K_{\varepsilon}$. Let us show that in this case $R_{n}$ satisfies assertions 1 and 2 of Theorem 2.5.

Assertion 1 of Monotonicity Lemma 2.10 implies that $R_{n}$ is actually a segment (as a preimage of a segment under a continuos monotonic map). Moreover, $\varepsilon=\alpha_{n}$ is a solution of the equation $(y-x)\left(\bar{E}_{\varepsilon}^{+}\right)=2 \pi n$ with respect to $\varepsilon$; according to assertion 2 of Lemma 2.10, $\alpha_{n}=O(1 / n)$, which proves assertion 2 of the theorem.

Let us estimate the length of the segment $R_{n}$, showing that $\left|R_{n}\right|=$ $O\left(e^{-C / \varepsilon}\right)$ for some $C>0$. Assertion 1 of the Shape Lemma 2.8 implies, that

$$
\begin{aligned}
\left|(y-x)\left(\bar{E}_{\varepsilon}^{+}\right)-(y-x)\left(\bar{E}_{\varepsilon}^{-}\right)\right| & \leq\left|x\left(\bar{E}_{\varepsilon}^{+}\right)-x\left(\bar{E}_{\varepsilon}^{-}\right)\right|+\left|y\left(\bar{E}_{\varepsilon}^{+}\right)-y\left(\bar{E}_{\varepsilon}^{-}\right)\right|= \\
& =\left|D_{\varepsilon}^{+}\right|+\left|D_{\varepsilon}^{-}\right|=O\left(e^{-C^{\prime} / \varepsilon}\right)=O\left(e^{-C n}\right)
\end{aligned}
$$

Together with assertion 1 of the Monotinicity Lemma and the mean value theorem it implies the required estimate for $\left|R_{n}\right|$. Hence assertions 1 and 2 of theorem 2.5 are proved for the selected $R_{n}$.

Let us show that if $\varepsilon$ does not belong to any $R_{n}$ then we are in the domain of case 1 from the previous section. This will imply assertion 3 of Theorem 2.5.

Proposition 2.13. Let $K_{\varepsilon} \cap \Delta=\varnothing$. Then the rotation number of $P_{\varepsilon}$ is an integer (to be more exact, $\rho(\varepsilon)=0(\bmod 2 \pi \mathbb{Z})$ ) and $P_{\varepsilon}$ has exactly two hyperbolic fixed points (one of them attracting and the other one repelling).

Proof. The original proof is given in [GI] (Proposition 1, p. 34). We reproduce it here with minor changes.

On the arc $S^{1} \backslash D_{\varepsilon}^{+}$, the graph $\gamma_{\varepsilon}$ of $P_{\varepsilon}$ has slope less than one. The endpoints of this graph lie on the left and the right boundaries of the rectangle 

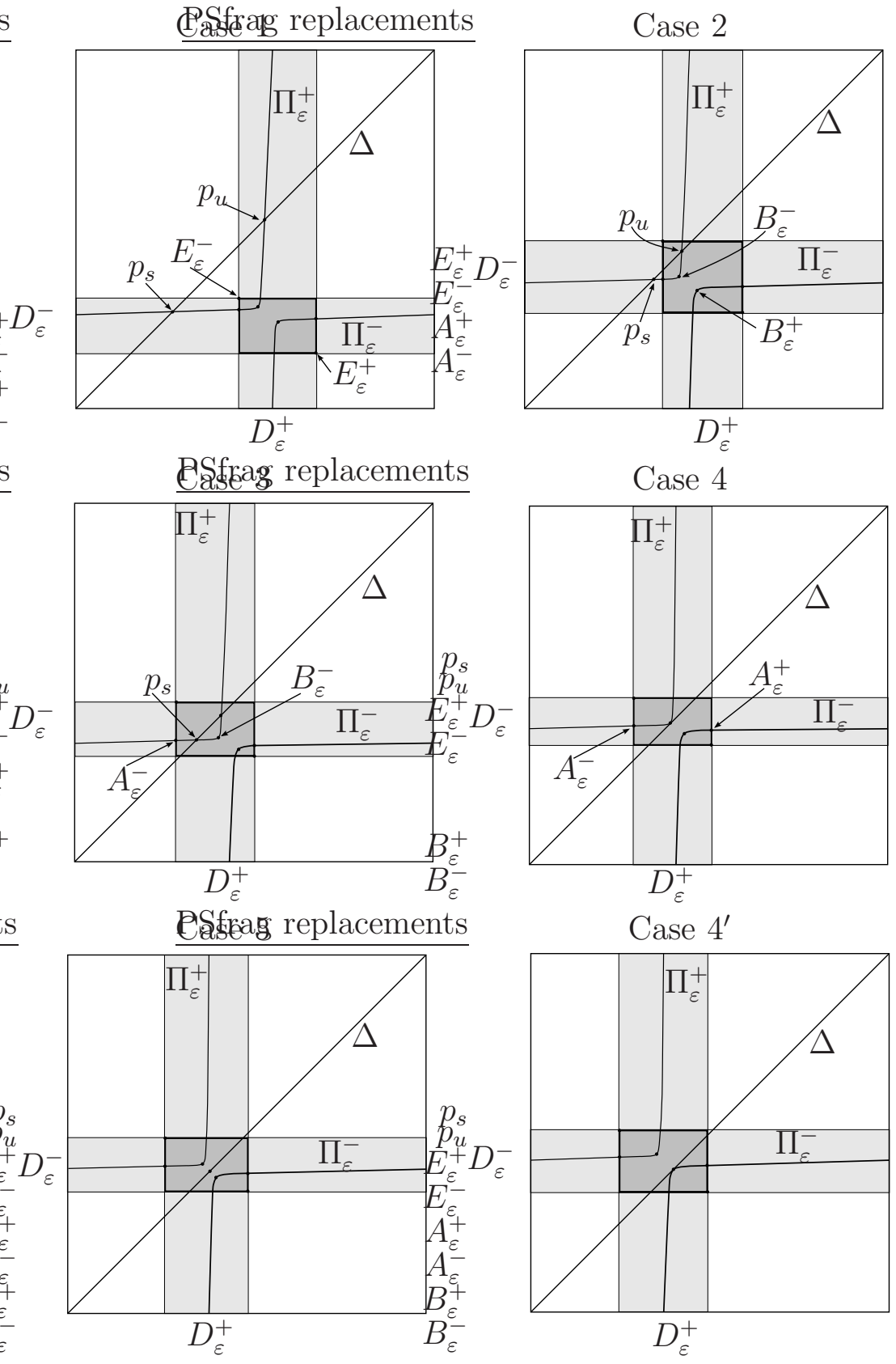

Figure 2.3: The graph of the Poincaré map. As $\varepsilon \rightarrow 0^{+}$cases $1,2,3,4$, 5 occur consecutively, then case 4 takes place again (denoted by "case $4^{\prime \prime \prime}$ ), then cases 3, 2, 1 (not shown), after which they repeat cyclically. 
$K_{\varepsilon}$ at the points $A_{\varepsilon}^{+}$and $A_{\varepsilon}^{-}$. If we connect these points by a segment inside $K_{\varepsilon}$, we obtain a closed curve $\gamma_{h}$ on $\mathbb{T}^{2}$. This curve has homotopy type $(1,0)$, because outside $K_{\varepsilon}$ its slope is less than 1 and it intersects each vertical circle $y=$ const at exactly one point. Consequently, $\gamma_{h}$ intersects $\Delta$ in some point. The Rolle lemma implies that this point is unique, because the slope of the curve is less than 1 . Let us denote it as $p_{s}$; it cannot lie in $K_{\varepsilon}$, because $\Delta$ does not intersect $K_{\varepsilon}$. Therefore, it is a fixed point of $P_{\varepsilon}$. It is stable because it lies outside $K_{\varepsilon}$.

Applying the same arguments to the inverse map $P_{\varepsilon}^{-1}$ (which is the Poincaré map after time inversion) we obtain the second (unstable) fixed point $p_{u}$, which also lies outside $K_{\varepsilon}$.

Let us prove assertion 4 of the Main Theorem 2.5. the existence and uniqueness of canard solutions. Let us define sets $\widetilde{C}_{n}^{-}$and $\widetilde{C}_{n}^{+}$in the following way:

$$
\begin{aligned}
& \widetilde{C}_{n}^{-}:=\left\{\varepsilon \in R_{n} \mid(y-x)\left(\bar{B}_{\varepsilon}^{-}\right)<2 \pi n<(y-x)\left(\bar{A}_{\varepsilon}^{-}\right)\right\}, \\
& \widetilde{C}_{n}^{+}:=\left\{\varepsilon \in R_{n} \mid(y-x)\left(\bar{A}_{\varepsilon}^{+}\right)<2 \pi n<(y-x)\left(\bar{B}_{\varepsilon}^{+}\right)\right\} .
\end{aligned}
$$

In other words, there are values of a parameter for which case 3, a stable fixed point inside $K_{\varepsilon}$, occurs. The Monotonicity and Convexity Lemmas imply that for $n$ big enough, the sets $\widetilde{C}_{n}^{ \pm}$are non-empty and open. Therefore, one can choose two open intervals $C_{n}^{ \pm} \subset \widetilde{C}_{n}^{ \pm}$for each $n$. By definition, $C_{n}^{-} \cup C_{n}^{+} \subset R_{n}$.

These intervals do not intersect each other: remark 2.9 implies that $(y-x)\left(B_{\varepsilon}^{-}\right)>(y-x)\left(B_{\varepsilon}^{+}\right)$(which means, $\left[(y-x)\left(B_{\varepsilon}^{-}\right),(y-x)\left(B_{\varepsilon}^{+}\right)\right\rangle \subset$ $\left.\left[E_{\varepsilon}^{+}, E_{\varepsilon}^{-}\right\rangle\right)$.

Let $\varepsilon \in C_{n}^{-}$(the case of $\varepsilon \in C_{n}^{+}$can be treated in a similar way). Take an arc $\lambda_{\varepsilon}$ of the graph $\gamma_{\varepsilon}$ with the endpoints $A_{\varepsilon}^{-}$and $B_{\varepsilon}^{-}$, which lies inside $K_{\varepsilon}$. By the definition of $C_{n}^{-}$, the endpoints of $\lambda_{\varepsilon}$ lie on different sides of $\Delta$ in $K_{\varepsilon}$, and thus it crosses $\Delta$. The intersection point belongs to $K_{\varepsilon}$. Convexity Lemma 2.12 implies that this point is unique and the slope of $\gamma_{\varepsilon}$ at this point is less than 1, so it corresponds to the stable fixed point of the Poincaré map.

Poincaré map is a well-defined diffeomorphism of a circle, so the existence of a stable fixed point implies the existence of an unstable fixed point.

Thus, theorem 2.5] is proved.

\section{Normalization}

In this and the next two sections, we prove Lemmas 2.8, 2.10 and 2.12. Lemma 2.8 is proved in subsection 4.1. Lemma 2.10 is proved in subsection 
4.3. Lemma 2.12 is proved in subsection 4.2 modulo an auxiliary Theorem 4.3. That theorem is proved in section 5.

We need two theorems (due to Guckenheimer and Ilyashenko) that describe normal forms of slow-fast systems on the two-torus.

\subsection{Nonlinear transition}

The following theorem describes slow-fast dynamics on the two-torus outside of some neighborhood of a slow curve.

Theorem 3.1 ([GI]). Consider a vector field on the cylinder $x \in S^{1}=\mathbb{R} / \mathbb{Z}$, $y \in \mathbb{R}$ defined by

$$
\dot{x}=f(x, y, \varepsilon), \quad \dot{y}=\varepsilon g(x, y, \varepsilon), \quad f>0, g>0
$$

Let $a, b \in \mathbb{R}, a<b$ and $\varepsilon>0$. The Poincaré map $P_{\varepsilon}^{[a, b\rangle}$ of a cross-section $\Gamma^{a}=\{y=a\}$ to $\Gamma^{b}=\{y=b\}$ has the form

$$
P_{\varepsilon}^{[a, b\rangle}(x)=G_{\varepsilon}^{1} \circ\left(G_{\varepsilon}^{2}(x)+T(\varepsilon)\right),
$$

where $T(\varepsilon) \rightarrow \infty$ as $\varepsilon \rightarrow O^{+}$and $G_{\varepsilon}^{1,2}$ are diffeomorphisms of a circle. $G_{\varepsilon}^{1,2} \rightarrow G_{1,2}$ as $\varepsilon \rightarrow 0^{+}$and $G_{1,2}$ are diffeomorphisms of a circle, as well.

In other words, the Poincaré map from one fixed vertical cross-section to another fixed vertical cross-section is a rotation by $T(\varepsilon)$ in properly selected coordinates on the preimage and image circles. $T(\varepsilon)$ tends to infinity as $\varepsilon \rightarrow 0^{+}$. It is important that corresponding coordinate maps have uniformly bounded derivatives as $\varepsilon \rightarrow 0^{+}$and tend to smooth maps.

Theorem 3.1 is proved in GI] (Theorem 2, p. 35).

\subsection{Normalization near the slow curve}

The following theorem shows that near the slow curve and outside of some neighborhood of the jump point system (2.1) is smoothly equivalent to a linear system.

Theorem 3.2 ([GI]). Consider the system

$$
\dot{x}=f(x, y, \varepsilon), \quad \dot{y}=\varepsilon g(x, y, \varepsilon), \quad g>0
$$

Let the corresponding fast system have a curve of nondegenerate fixed points (which is the nondegenerate slow curve). Then for small $\varepsilon>0$ near this curve (and outside any small fixed neighborhood of jump points) the system is smoothly orbitally equivalent to the family 


$$
\dot{x}=a(y, \varepsilon) x, \quad \dot{y}=\varepsilon
$$

The proof of this theorem can be found in [GI] (Theorem 3, p. 38). The Fenichel theorem (see [F], and also [A1] and [A2]) implies that in some neighborhood of the stable (unstable) parts of the slow curve $M^{-}\left(M^{+}\right)$ there exists a smooth invariant manifold $S_{\varepsilon}^{-}\left(S_{\varepsilon}^{+}\right)$, which can be seen as the graph of the function $x=s^{-}(y, \varepsilon)\left(x=s^{+}(y, \varepsilon)\right.$ resp.). Variational equations imply that the function $a(y, \varepsilon)$ in Theorem 3.2 has the form:

$$
a(y, \varepsilon)=f_{x}^{\prime}(s(y, \varepsilon), y, \varepsilon)
$$

Remark 3.3. True slow curves $S_{\varepsilon}^{ \pm}$are non-unique. However, the distance between any such curves is of order $O\left(e^{-C / \varepsilon}\right)$ (thus they are exponentially close to each other) and we can choose any of them for our analysis.

\subsection{Rough estimate of the derivative of the Poincaré map}

Let us prove a preliminary estimate for the derivative of the Poincaré map.

Remark 3.4. Here and below we will use letter $C$ (without indicies) to denote (generally different) positive constants that do not depend on $\varepsilon$.

Lemma 3.5. Consider the slow-fast system

$$
\dot{x}=f(x, y, \varepsilon), \quad \dot{y}=\varepsilon, \quad(x, y) \in S^{1} \times \mathbb{R}
$$

For $\varepsilon>0$ small enough the following estimate for Poincaré map $P_{\varepsilon}^{[a, b\rangle}: \Gamma^{a} \rightarrow$ $\Gamma^{b}$ holds:

$$
\left|\ln \left(P_{\varepsilon}^{[a, b\rangle}(x)\right)^{\prime}\right|<C \frac{b-a}{\varepsilon},
$$

Proof. Let $x=x\left(y ; x_{0}, \varepsilon\right)$ be the phase curve that contains the point $\left(x_{0}, a\right)$ for given $\varepsilon$. As $f_{x}^{\prime}$ is bounded (from above and from below) on the two-torus, variational equations imply that

$$
\left(P_{\varepsilon}^{[a, b\rangle}\left(x_{0}\right)\right)^{\prime}=\exp \left(\frac{1}{\varepsilon} \int_{a}^{b} f_{x}^{\prime}\left(x\left(y ; x_{0}, \varepsilon\right), y, \varepsilon\right) d y\right)<e^{\frac{C}{\varepsilon}(b-a)}
$$

The same estimate holds for the inverse map. Applying the logarithm we obtain the required estimate.

This lemma will be used in the neighborhood of a jump point. More precise estimates of the derivative of the Poincaré map near a jump point are presented in subsection 5.1 


\section{Properties of the Poincaré map}

In this section and two sections that follow we prove Lemmas $2.8,2.10$ and 2.12 .

\subsection{Distortion: proof of Lemma 2.8}

Recall the notation:

$$
\begin{gathered}
\alpha^{ \pm}=\tau^{ \pm} \pm \delta_{ \pm} \\
\Gamma^{ \pm}=\left\{y=\alpha^{ \pm}\right\} \\
J^{+}:=\left\{(x, y) \in \Gamma^{+} \mid x \in\left[\sigma^{+}, \pi\right]\right\}, \quad J^{-}:=\left\{(x, y) \in \Gamma^{-} \mid x \in\left[-\pi, \sigma^{-}\right]\right\}
\end{gathered}
$$

Denote $P_{\varepsilon}^{\left[-\pi, \alpha^{+}\right\rangle}$as $Q_{\varepsilon}$ for brevity. Recall that $D_{\varepsilon}^{+}=\left(Q_{\varepsilon}\right)^{-1}\left(J^{+}\right)$.

Proposition 4.1. For any $\delta_{+}$small enough there exist constants $c_{1,2}\left(\delta_{+}\right)>0$ such that the following estimates hold:

1. $\left|D_{\varepsilon}^{+}\right|=O\left(e^{-c_{1}\left(\delta_{+}\right) / \varepsilon}\right)$;

2. $Q_{\varepsilon}^{\prime} \leq C e^{c_{2}\left(\delta_{+}\right) / \varepsilon}$.

Moreover, $c_{1,2}\left(\delta_{+}\right) \rightarrow 0^{+}$as $\delta_{+} \rightarrow 0^{+}$.

Remark 4.2. Let us denote the Poincaré map from $\Gamma^{-}$to $\Gamma$ (in forward time) as $\widetilde{Q}_{\varepsilon}$. For the system with the reversed time similar assertions can be made for $\widetilde{Q}_{\varepsilon}$ and some other constants $c_{1,2}$ :

1. $\left|D_{\varepsilon}^{-}\right|=O\left(e^{-c_{1}\left(\delta_{-}\right) / \varepsilon}\right)$;

2. $\left(\widetilde{Q}_{\varepsilon}^{-1}\right)^{\prime} \leq C e^{c_{2}\left(\delta_{-}\right) / \varepsilon}$.

Moreover, $c_{1,2}\left(\delta_{-}\right) \rightarrow 0^{+}$as $\delta_{-} \rightarrow 0^{+}$.

It can be proven by the same arguments, applied to the system with the reversed time.

Proof of proposition 4.1. In order to prove the first assertion let us estimate the derivative of $Q_{\varepsilon}$ on the segment $J^{+}$. Consider cross-sections

$$
\Gamma_{1}=\left\{y=\tau^{+}+\delta_{+}^{2}\right\}, \quad \Gamma_{2}=\left\{y=\tau^{+}-\delta_{+}^{2}\right\} .
$$

Denote a segment $\left[\tau^{+}+\delta_{+}^{2}, \tau^{-}-\delta_{-}^{2}\right]$ by $\Sigma$ and let $S_{\varepsilon}^{-}$and $S_{\varepsilon}^{+}$be the stable and unstable parts of the true slow curve over the segment $\Sigma$, resp. Also, denote the segment $\left[\tau^{+}+\delta_{+}^{2}, \tau^{+}+\delta_{+}\right]$by $\Sigma^{+}$.

The map $Q_{\varepsilon}^{-1}$ can be presented as a composition of the following Poincaré maps: 


$$
\begin{gathered}
Q_{\varepsilon}^{-1}: \Gamma^{+} \stackrel{Q_{1}}{\rightarrow} \Gamma_{1} \stackrel{Q_{2}}{\rightarrow} \Gamma_{2} \stackrel{Q_{3}}{\rightarrow} \Gamma \\
Q_{\varepsilon}^{-1}=Q_{3} \circ Q_{2} \circ Q_{1}
\end{gathered}
$$

We will use normal forms to analyse maps $Q_{1}$ and $Q_{3}$ and Lemma 3.5 to estimate the derivative of $Q_{2}$.

\subsubsection{Linear contraction: neighborhood of the true slow curve}

Let us prove the following estimate:

$$
\left.Q_{1}^{\prime}\right|_{J^{+}} \leq C_{1} \exp \left(-\frac{C \delta_{+}^{3 / 2}}{\varepsilon}\right)
$$

where $C, C_{1}$ are positive constants that do not depend on $\delta_{+}$.

When trajectories that cross $J^{+}$are traced back in time from $\Gamma^{+}$to $\Gamma^{1}$, we see that the amount of time they spend outside of some neighborhood of $\mathrm{M}^{-}$ is uniformly bounded from above. Thus we can use the normal form (3.4) to study the map $Q_{1}$ (see Theorem 3.2).

We shift coordinates by moving the origin to the jump point $G^{+}=$ $\left(\tau^{+}, \sigma^{+}\right)$:

$$
x_{1}=x-\sigma^{+}, \quad y_{1}=y-\tau^{+}
$$

We will apply normal form (3.4) over the segment $\Sigma^{+}$for small $\delta_{ \pm}$. (Note that $\Sigma^{+}$does not depend on $\varepsilon$.) Let us show that for $y_{1} \in \Sigma^{+}$the function $a\left(y_{1}, \varepsilon\right)$ has the following form:

$$
a\left(y_{1}, \varepsilon\right)=(C+o(1)) \sqrt{y_{1}}+O_{\delta_{+}}(\varepsilon),
$$

where $o(1)$ is a function of $y_{1}$ and $\varepsilon$ which tends to 0 uniformly as $\delta_{+} \rightarrow 0$ and $O_{\delta_{+}}(\varepsilon)$ is of order $\varepsilon$ for any fixed $\delta_{+}$. Indeed, from nondegenericity assumptions (2.5) and the implicit function theorem, it follows that $M^{+}$can be presented as a graph of the function

$$
x=s^{+}\left(y_{1}\right)=(C+o(1)) \sqrt{y_{1}} .
$$

Thus the true slow curve is a graph of the function

$$
x=s^{+}\left(y_{1}, \varepsilon\right)=(C+o(1)) \sqrt{y_{1}}+O_{\delta_{+}}(\varepsilon) .
$$

Substitute (4.6) into the expression for $a\left(y_{1}, \varepsilon\right)$ (see (3.5)). We obtain: 


$$
\begin{aligned}
& a\left(y_{1}, \varepsilon\right)=f_{x}^{\prime}\left(s\left(y_{1}, \varepsilon\right), y, \varepsilon\right)= \\
& \quad=f_{x x}^{\prime \prime}(0,0,0)\left(s\left(y_{1}\right)+O_{\delta_{+}}(\varepsilon)\right)+O(|y|+\varepsilon)= \\
& =(C+o(1)) \sqrt{y_{1}}+O_{\delta_{+}}(\varepsilon)
\end{aligned}
$$

where $\left.f_{x x}^{\prime \prime}\right|_{0}>0$ according to (2.5).

From the normal form (3.4) it follows that the map $Q_{1}$ is linear in normalized chart. To simplify the notation, let $\delta=\delta_{+}$. Substituting expression (4.7) for $a(y, \varepsilon)$ into (3.4) and integrating, we have:

$$
Q_{1}\left(x_{1}\right)=\Lambda(\varepsilon) x_{1}, \quad \Lambda(\varepsilon)=e^{-\frac{\delta^{3 / 2}}{\varepsilon}\left(C+o(1)+O_{\delta}(\varepsilon)\right)}<e^{-\frac{\delta^{3 / 2}}{\varepsilon}\left(\frac{3}{4} C\right)}
$$

The normalizing map that we applied to obtain the normal form has its derivative bounded away from $\infty$ and 0 . Thus in the original coordinates the right-hand part of (4.2) can be taken to be a constant times $\Lambda(\varepsilon)$.

\subsubsection{Neighborhood of the jump point}

Let us show that for $\delta$ small enough the expansion of the phase curves, which is possible near the jump point (during the transition from $\Gamma_{1}$ to $\Gamma_{2}$ ) is much less than their contraction that was accumulated during the transition near the slow curve. Indeed, Lemma 3.5 yields the following estimate:

$$
Q_{2}^{\prime} \leq e^{C \delta^{2} / \varepsilon} \ll \Lambda^{-1}(\varepsilon) .
$$

\subsubsection{Estimating the derivative of $Q_{\varepsilon}$}

Map $Q_{3}$ is a rotation in rectifying charts. Its derivative is bounded uniformly in $\varepsilon$.

Using the chain rule, we get:

$$
\left.Q_{\varepsilon}^{-1}\right|_{J^{+}} ^{\prime}=O\left(e^{-C / \varepsilon}\right), \quad C=O\left(\delta_{+}^{3 / 2}\right)
$$

Thus,

$$
\left|D_{\varepsilon}^{+}\right|=O\left(e^{-C / \varepsilon}\right), \quad C=O\left(\delta_{+}^{3 / 2}\right)
$$

Assertion 1 of Proposition 4.1 is proved. Due to Remark 4.2 this proves assertion 1 of Lemma 2.8 .

Let us prove assertion 2 of Proposition 4.1. Consider the map $Q_{\varepsilon}$ : 


$$
Q_{\varepsilon}=\left(Q_{1}\right)^{-1} \circ\left(Q_{2}\right)^{-1} \circ\left(Q_{3}\right)^{-1}
$$

The derivative of $\left(Q_{3}\right)^{-1}$ can be estimated from above by Theorem 3.1 and so is bounded by a constant. The time of the transition from $\Gamma_{2}$ to $\Gamma^{+}$is no greater than $2 \delta_{+}$. Applying Lemma 3.5 to the map $\left(Q_{1}\right)^{-1} \circ\left(Q_{2}\right)^{-1}$, we obtain the desired estimate for its derivative. Along with the chain rule, it completes the proof.

Let us prove assertion 4 of Lemma 2.8. We present $P_{\varepsilon}$ as a composition:

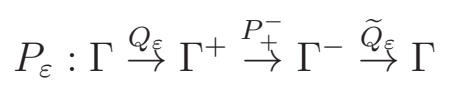

By definition, the trajectories that start outside of $D_{\varepsilon}^{+}$, intersect $\Gamma^{+}$outside of segment $J^{+}$:

$$
Q_{\varepsilon}\left(\Gamma \backslash D_{\varepsilon}^{+}\right)=\Gamma^{+} \backslash J^{+}
$$

Note that outside of any neighborhood of the slow curve the function $f$ is bounded away from zero. Therefore it will take time of order $O(1)$ for the trajectory that crosses $\Gamma^{+} \backslash J^{+}$to reach certain neighborhood of the stable part of the slow curve $M^{-}$. Due to the normal form (3.4), the derivative of the map $P_{+}^{-}$satisfies the following estimate:

$$
\left.\left(P_{+}^{-}\right)^{\prime}\right|_{\Gamma^{+} \backslash J^{+}}<e^{-C / \varepsilon},
$$

where $C$ is bounded away from 0 as $\delta_{ \pm} \rightarrow 0$.

Therefore, the image of segment $\Gamma^{+} \backslash J^{+}$has exponentially small length and intersects the true slow curve. Thus $P_{+}^{-}\left(\Gamma^{+} \backslash J^{+}\right) \subset J^{-}$for $\varepsilon>0$ small enough. We have:

$$
P_{\varepsilon}\left(\Gamma \backslash D_{\varepsilon}^{+}\right) \subset D_{\varepsilon}^{-},
$$

which implies assertion 4 of Lemma 2.8 ,

Let us prove assertion 2 of Lemma 2.8 (assertion 3 can be proven by the same arguments applied to the system with reversed time). From estimate (4.15), it follows that for a trajectory that crosses $\Gamma \backslash D_{\varepsilon}^{+}$, the derivative of the map $P_{+}^{-}$is less than 1 and, moreover, it is exponentially small as $\varepsilon \rightarrow 0^{+}$. Similar to (4.10), one can show that the derivative of $\widetilde{Q}_{\varepsilon}$ is exponentially small on the segment $J^{-}$. Derivative of the map $Q_{\varepsilon}$ is less than $O\left(e^{c\left(\delta_{+}\right) / \varepsilon}\right)$ according to assertion 2 of Proposition 4.1 and $c\left(\delta_{+}\right)$can be considered small enough for an appropriate choice of $\delta_{+}$. Therefore, any possible expansion by $Q_{\varepsilon}$ is controlled by the exponential contraction $P_{+}^{-}$, and the derivative of $P_{\varepsilon}$ is exponentially small.

Lemma 2.8 is proven. 


\subsection{Convexity: proof of Lemma 2.12}

In this section Lemma, 2.12 will be proved. Recall that we have defined the set $U$ of initial conditions $u \in \Gamma$, for which

$$
P_{\varepsilon}^{\prime}(u) \in[1 / 2,2] .
$$

We will show that $U$ consists of two arcs of the circle $\Gamma$ : on one arc the derviative of the Poincaré map increases (the graph $\gamma_{\varepsilon}$ is convex), and on the other one it decreases. All along the proof we will assume that the initial condition of every trajectory considered belongs to $U$.

\subsubsection{Heuristic motivation}

As Lemma 2.8 shows, outside of the segment $D_{\varepsilon}^{+}$the derivative of the Poincaré map $P_{\varepsilon}$ is exponentially small. Therefore, the set $U$ lies in $D_{\varepsilon}^{+}$ and corresponding trajectories cross $J^{+}$.

After intersecting $J^{+}$any such trajectory spends some time near the unstable part of the slow curve $M^{+}$. Then it jumps either up or down, leaves the neighborhood of $M^{+}$, and approaches the neighborhood of the stable part of true slow curve $M^{-}$in bounded time. Calculating the derivative of the Poincaré map, one easily sees that it increases while the trajectory spends time near $M^{+}$and decreases while the trajectory passes along $M^{-}$(which follows from the normal form (3.4)). We do not consider trajectories which spend too much time near either $M^{+}$or $M^{-}$: the derivative of the Poincaré map for such trajectories is either too big or too small. Trajectories we are interseted in jump "somewhere in between" and spend comparable amount of time near $M^{+}$and $M^{-}$. (Later we will give rigorous definitions for this.)

After the jump (either upwards or downwards), those trajectories cross $J^{-}$, jump near the point $G^{-}$and cross $\Gamma$ in a point that belongs to $D_{\varepsilon}^{-}$.

Let us extend the true slow curve $M_{\varepsilon}^{+}$in reverse time and denote the point of its intersection with the cross-section $\Gamma$ by $u_{0}$. Obviously, $u_{0} \in D_{\varepsilon}^{+}$. Consider trajectories starting from points $u \in D_{\varepsilon}^{+}$that lie lower than $u_{0}$. When $u$ tends to $u_{0}$ from below, the corresponding trajectory tends to $S_{\varepsilon}^{+}$ and thus it spends more time near $M^{+}$and less time near $M^{-}$. Hence when $u$ increases, the derivative of the Poincaré map increases too. When $u$ coincides with $u_{0}$, the derivative reaches its maximum value, because the corresponding trajectory coincides with the true slow curve $S_{\varepsilon}^{+}$. After that, as $\mathrm{u}$ increases, the derivative of the Poincaré map will decrease for similar reasons: the trajectory spends less time near $M^{+}$, and more time near $M^{-}$.

The foregoing analysis shows that these naive arguments do work. 


\subsubsection{The strategy}

We will use the same method to deal with $P_{\varepsilon}$ as we used to prove Lemma 2.8. we decompose the map $P_{\varepsilon}$ into the composition of several Poincaré maps. By analyzing the dynamics near $M^{+}$and $M^{-}$, we will show that the trajectories for which the derivative of the Poincare map is close to 1 leave the neighborhood of $M^{+}$near some fixed cross-section $y=y_{+}^{-}$. Afterwards, using variational equations, we will estimate the second derivative of the Poincaré map.

To proceed with this strategy we need additional information describing the dynamics near the jump point.

Theorem 4.3. For some constant $\lambda>0$ and arbitrarily small $\delta_{+}$, there exists a $C^{+}\left(\delta_{+}\right)$, such that, for arbitrary $x \in D_{\varepsilon}^{+}$, the following representation of the derivative of the Poincaré map $R_{\varepsilon}=Q_{\varepsilon}^{-1}=P_{\varepsilon}^{\left\langle-\pi, \alpha^{+}\right]}$holds:

$$
\ln R_{\varepsilon}^{\prime}(x)=\frac{C^{+}\left(\delta_{+}\right)+O\left(\varepsilon^{\lambda}\right)}{\varepsilon},
$$

where $C^{+}\left(\delta_{+}\right)<0$ is continuous and tends monotonically to zero as $\delta_{+} \rightarrow 0^{+}$

Remark 4.4. A similar expression (with another constant $C^{-}\left(\delta_{-}\right)<0$, which tends to 0 monotonically as $\left.\delta_{-} \rightarrow 0\right)$ holds for $\widetilde{Q}_{\varepsilon}=P_{\varepsilon}^{\left[\alpha^{-}, \pi\right\rangle}$.

Theorem 4.3 is proved in section 5 .

We denote the normalizing charts near unstable and stable parts of the true slow curve by $\left(x_{+}, y\right)$ and $\left(x_{-}, y\right)$, resp. Define $U^{ \pm}:=\left\{\left|x_{ \pm}\right|<b\right\}$ for some $b>0$. Let us first deal with the case when the trajectory jumps from $S^{+}$ to $S^{-}$in the negative direction (down). Suppose that the trajectory under consideration leaves the neighborhood $U^{+}$when $y=y^{+}$and reaches $U^{-}$ when $y=y^{-}$. Obviosly, $y^{-}=y^{+}+O(\varepsilon)$ (since outside of $U^{ \pm}$, the function $f$ is bounded away from 0$)$. We assume that in the charts $\left(x_{ \pm}, y\right)$, system (2.1) has the following form:

$$
\dot{x}_{ \pm}=a^{ \pm}(y, \varepsilon) x_{ \pm}, \quad \dot{y}=\varepsilon
$$

Let us define

$$
\Phi^{ \pm}(y, \varepsilon)=\int_{\alpha^{ \pm}}^{y} a^{ \pm}(v, \varepsilon) d v, \quad \Phi^{ \pm}(y)=\Phi^{ \pm}(y, 0)
$$

Function $\Phi^{-}$(resp. $\left.\Phi^{+}\right)$is equal to the logarithmic derivative of the corresponding Poincaré map in the normalizing chart. In other words, they estimate contraction (expansion) of trajectories, accumulated during the transition near stable (resp. unstable) part of the slow curve. Since $a^{+}(y, \varepsilon)>0$ 
and $a^{-}(y, \varepsilon)<0$, and $\alpha^{+}<y<\alpha^{-}$, it follows that $\Phi^{ \pm}(y, \varepsilon)>0$, and $\Phi^{+}$ increases with $y$, while $\Phi^{-}$decreases. Let $y=y_{+}^{-}$be the root of the equation:

$$
\Phi^{+}(y, 0)-\Phi^{-}(y, 0)=0
$$

In order to satisfy (4.17) it is neccessary that $y^{ \pm}$is close to $y_{+}^{-}$. Otherwise, either attraction or repulson will dominate in $P_{\varepsilon}^{\prime}$, though (4.17) demands them to annihilate. The next Lemma formalizes this heuristic arguments.

Lemma 4.5. There exists $\lambda>0$, such that for an appropriate choice of a small $\delta_{ \pm}$and for any trajectory with initial condition in $U$, the following estimates hold:

$$
\left|y_{+}^{-}-y^{+}\right|=O\left(\varepsilon^{\lambda}\right), \quad\left|y_{+}^{-}-y^{-}\right|=O\left(\varepsilon^{\lambda}\right) .
$$

These two estimates are equivalent because $\left|y^{+}-y^{-}\right|=O(\varepsilon)$.

Proof. Let us decompose the Poincaré map $P_{\varepsilon}$ :

$$
\begin{gathered}
P_{\varepsilon}: \Gamma \stackrel{Q_{\varepsilon}}{\rightarrow} \Gamma^{+} \stackrel{P_{+}^{-}}{\rightarrow} \Gamma^{-} \stackrel{\widetilde{Q}_{\varepsilon}}{\rightarrow} \Gamma \\
P_{\varepsilon}=\widetilde{Q}_{\varepsilon} \circ P_{+}^{-} \circ Q_{\varepsilon},
\end{gathered}
$$

where $Q_{\varepsilon}=R_{\varepsilon}^{-1}$. The chain rule implies:

$$
\ln P_{\varepsilon}^{\prime}=\ln \widetilde{Q}_{\varepsilon}^{\prime} \circ P_{+}^{-} \circ Q_{\varepsilon}+\ln \left(P_{+}^{-}\right)^{\prime} \circ Q_{\varepsilon}+\ln Q_{\varepsilon}^{\prime}
$$

First, we estimate the second term of this sum from below. Theorem 3.2 and variational equations imply:

$$
\begin{aligned}
\left|\ln \left(P_{+}^{-}\right)^{\prime}\right| & =\left|\frac{1}{\varepsilon}\left(\Phi^{+}\left(y^{+}, \varepsilon\right)-\Phi^{-}\left(y^{-}, \varepsilon\right)\right)\right|= \\
& =\left|\frac{1}{\varepsilon}\left(\Phi^{+}\left(y^{+}\right)-\Phi^{-}\left(y^{-}\right)\right)\right|+O(1)= \\
& =\frac{1}{\varepsilon}\left|\Phi^{+}\left(y^{+}\right)-\Phi^{+}\left(y_{+}^{-}\right)-\Phi^{-}\left(y^{-}\right)+\Phi^{-}\left(y_{+}^{-}\right)\right|+O(1)= \\
& =\frac{1}{\varepsilon}\left|-\int_{y^{+}}^{y_{+}^{-}} a^{+}(y, 0) d y+\int_{y^{-}}^{y_{+}^{-}} a^{-}(y, 0) d y\right|+O(1)> \\
& >c \frac{\left|y^{+}-y_{+}^{-}\right|}{\varepsilon},
\end{aligned}
$$

where $c=\frac{1}{2} \min _{\Sigma}\left(a^{+}(y, 0)-a^{-}(y, 0)\right)>0$ since $a^{+}(y, 0)>0$ and $a^{-}(y, \varepsilon)<0$. 
According to theorem 4.3,

$$
\ln Q_{\varepsilon}^{\prime}=\frac{C_{+}\left(\delta_{+}\right)+O\left(\varepsilon^{\lambda}\right)}{\varepsilon}, \quad \ln \widetilde{Q}_{\varepsilon}^{\prime}=\frac{-C_{-}\left(\delta_{-}\right)+O\left(\varepsilon^{\lambda}\right)}{\varepsilon}
$$

It follows from the asymptotic behaviour of $C_{ \pm}$that one can find small $\delta_{ \pm}$ such that $C_{+}\left(\delta_{+}\right)=C_{-}\left(\delta_{-}\right)$. For such $\delta_{ \pm}$, condition (4.17) implies:

$$
\ln 2 \geq\left|\ln Q_{\varepsilon}^{\prime}+\ln P_{+}^{-}+\ln \widetilde{Q}_{\varepsilon}^{\prime}\right|>c \frac{\left|y^{+}-y_{+}^{-}\right|+O\left(\varepsilon^{\lambda}\right)}{\varepsilon} .
$$

Therefore,

$$
\begin{gathered}
\left|y^{+}-y_{+}^{-}\right|=O(\varepsilon)+O\left(\varepsilon^{\lambda}\right)=O\left(\varepsilon^{\lambda}\right), \quad \lambda>0 \\
y_{+}^{-}=y^{+}+\varepsilon^{\lambda} k_{1}\left(y^{+}, \varepsilon\right),
\end{gathered}
$$

where $k_{1}\left(y^{+}, \varepsilon\right)$ is a smooth function.

Let us take the derivative of (4.23):

$$
\frac{d}{d u} \log P_{\varepsilon}^{\prime}=\frac{d}{d u} \log \widetilde{Q}_{\varepsilon}^{\prime} \circ P_{+}^{-} \circ Q_{\varepsilon}(u)+\frac{d}{d u} \log \left(P_{+}^{-}\right)^{\prime} \circ Q_{\varepsilon}(u)+\frac{d}{d u} \log Q_{\varepsilon}^{\prime}(u)
$$

We will show shat the sign of the lograthmic derivative of the Poincaré map depends only on the sign of the second term in this expression. The other two terms's influence can be estimated from above:

$$
\begin{aligned}
& \left|\frac{d}{d u} \log Q_{\varepsilon}^{\prime}(u)\right|<C \cdot \exp \left(\frac{\delta_{+}^{3 / 2}}{\varepsilon}\right) \\
& \left|\frac{d}{d u} \log \widetilde{Q}_{\varepsilon}^{\prime}(u)\right|<C \cdot \exp \left(\frac{\delta_{-}^{3 / 2}}{\varepsilon}\right)
\end{aligned}
$$

Proof of these estimates can be found in [GI], p. 44. It only requires the fact that $f$ and its derivative are bounded and that assertion 2 of Proposition 4.1 holds. Once it is assured, the proof from [GI] works without any changes.

Proposition 4.6. Suppose $\varepsilon$ and $u$ such that (4.17) holds. Let

$$
I=2 \Phi^{+}\left(y_{+}^{-}\right)=\Phi^{+}\left(y_{+}^{-}\right)+\Phi^{-}\left(y_{+}^{-}\right) .
$$

Then assuming that $\varepsilon$ is sufficiently small, we have the following estimate:

$$
\left|\frac{d}{d u} \ln \left(P_{+}^{-}\right)^{\prime} \circ Q_{\varepsilon}(u)\right|>\exp \frac{I}{5 \varepsilon}
$$


It is easy to see that provided (4.31) the second term of the sum (4.29) dominates. Thus, when trajectories jump down, the whole expression (4.29) is positive. Similar arguments show that the derivative is negative if trajectories jump up. The rest of the section is devoted to the proof of Proposition 4.6, which thus concludes the proof of Lemma 2.12.

Proof. Let us consider the normalizing chart $\xi$ on the cross-section $J^{+}$near the slow curve, and the normalizing chart $\eta$ on the cross section $J^{-}$. For the trajectory that intersects $J^{+}$in $\xi$, we denote its intersection with $J^{-}$by $\eta(\xi)$. In the case we are considering (when trajectories jump down) $\xi$ is negative. The function $\xi \mapsto \eta(\xi)$ defines the Poincaré map $P_{+}^{-}$in normalizing charts. As direct calculations show (see [GI], p. 43), we have:

$$
\eta(\xi)=-\xi \exp \left(\frac{\Phi^{+}\left(y^{+}, \varepsilon\right)-\Phi^{-}\left(y^{-}, \varepsilon\right)}{\varepsilon}\right)
$$

However, according to (4.22):

$$
\begin{gathered}
\Phi^{+}\left(y^{+}, \varepsilon\right)-\Phi^{-}\left(y^{-}, \varepsilon\right)-\left(2 \Phi^{+}\left(y^{-}, \varepsilon\right)-I\right)= \\
=\Phi^{+}\left(y^{+}, \varepsilon\right)-\Phi^{-}\left(y^{-}, \varepsilon\right)-\left(2 \Phi^{+}\left(y^{-}, \varepsilon\right)-\Phi^{+}\left(y_{+}^{-}\right)-\Phi^{-}\left(y_{+}^{-}\right)\right)= \\
=\Phi^{+}\left(y^{+}\right)-\Phi^{-}\left(y^{+}\right)-\left(2 \Phi^{+}\left(y^{+}\right)-\Phi^{+}\left(y_{+}^{-}\right)-\Phi^{-}\left(y_{+}^{-}\right)\right)+O(\varepsilon)= \\
=\left(-\Phi^{-}\left(y^{+}\right)+\Phi^{-}\left(y_{+}^{-}\right)\right)+\left(-\Phi^{+}\left(y^{+}\right)+\Phi^{+}\left(y_{+}^{-}\right)\right)+O(\varepsilon)= \\
=\varepsilon^{\lambda} k_{2}\left(y^{+}, \varepsilon\right),
\end{gathered}
$$

where $k_{2}\left(y^{+}, \varepsilon\right)$ is a smooth function.

On the other hand, by definition of $y^{+}$,

$$
\exp \left(\frac{\Phi^{+}\left(y^{+}, \varepsilon\right)}{\varepsilon}\right)=-\frac{b}{\xi} .
$$

The minus sign is due to $\xi<0$ in the case we are considering. It will be opposite in the other case (when trajectory jumps up).

Substituting $\Phi^{+}\left(y^{+}, \varepsilon\right)-\Phi^{-}\left(y^{-}, \varepsilon\right)$ into (4.32) with the expression which follows from (4.33), and using (4.34), we obtain:

$$
\eta(\xi)=-\frac{b^{2}}{\xi} \exp \left(\frac{-I+\varepsilon^{\lambda} k\left(y^{+}, \varepsilon\right)}{\varepsilon}\right),
$$

where $k\left(y^{+}, \varepsilon\right)$ is a smooth function.

Let us show that $\eta(\xi)$ behaves like - const $\cdot \xi^{-1}$, i.e. it is convex for negative $\xi$.

Equation (4.34) implies that 


$$
\Phi^{+}\left(y^{+}, \varepsilon\right)=\varepsilon(\ln b-\ln (-\xi)) .
$$

The function $\Phi^{+}\left(y^{+}, \varepsilon\right)$ is strictly $y^{+}$-monotonic due to nondegeniricity condition (2.3). Thus there exists an inverse function. Denote it by $z_{\varepsilon}$ :

$$
z_{\varepsilon}\left(\Phi^{+}\left(y^{+}, \varepsilon\right)\right) \equiv y^{+}
$$

Then

$$
y^{+}=z_{\varepsilon}(\varepsilon(\ln b-\ln (-\xi))) .
$$

We substitute (4.38) into (4.35) and take the derivative:

$$
\begin{gathered}
\eta^{\prime}(\xi)=b^{2} e^{-I / \varepsilon} \frac{1}{\xi^{2}} e^{\left(\varepsilon^{\lambda-1} k\left(y^{+}, \varepsilon\right)\right)}\left(k^{\prime} z_{\varepsilon}^{\prime} \varepsilon^{\lambda}+1\right), \\
\ln \eta^{\prime}(\xi)=2 \ln b-\frac{I}{\varepsilon}-2 \ln (-\xi)+\varepsilon^{\lambda-1} k\left(y^{+}, \varepsilon\right)+\ln \left(k^{\prime} z_{\varepsilon}^{\prime} \varepsilon^{\lambda}+1\right), \\
\frac{d}{d \xi} \ln \eta^{\prime}(\xi)=-\frac{2}{\xi}-\varepsilon^{\lambda} k^{\prime} z^{\prime} \frac{1}{\xi}-\frac{\varepsilon^{\lambda+1}}{\xi} \frac{k^{\prime \prime}\left(z^{\prime}\right)^{2}+k^{\prime} z^{\prime \prime}}{1+k^{\prime} z^{\prime} \varepsilon^{\lambda}}>\frac{1}{-\xi} .
\end{gathered}
$$

Obviously,

$$
\Phi^{+}\left(y^{+}\right)>\frac{I}{3}
$$

By (4.34), we have

$$
-\xi=b \exp \frac{-\Phi^{+}\left(y^{+}, \varepsilon\right)}{\varepsilon}
$$

Hence,

$$
\frac{d}{d \xi} \log \eta^{\prime}(\xi)>-\frac{1}{\xi}>\frac{1}{b} \exp \frac{I}{3 \varepsilon}
$$

The transition from the normalizing charts back to the initial charts may only multiply the derivative by a bounded function and will not considerably affect the exponential estimate we just obtained:

$$
\frac{d}{d u} \ln \left(P_{+}^{-}\right)^{\prime}>\frac{1}{b} \exp \frac{I}{4 \varepsilon}
$$

The chain rule and the estimate from assertion 2 of proposition 4.1 imply that

$$
\begin{aligned}
\frac{d}{d u} \ln \left(P_{+}^{-}\right)^{\prime} \circ Q_{\varepsilon}(u)=\frac{d}{d x}\left(\ln \left(P_{+}^{-}\right)^{\prime}\right) \cdot Q_{\varepsilon}^{\prime}(u)> & \\
& >\frac{1}{b} \exp \left(\frac{I+o(1)}{4 \varepsilon}\right)>\frac{1}{b} \exp \left(\frac{I}{5 \varepsilon}\right) .
\end{aligned}
$$


For trajectories that jump up, the corresponding estimate takes the form:

$$
\frac{d}{d u} \ln \left(P_{+}^{-}\right)^{\prime} \circ Q_{\varepsilon}(u)<-\frac{1}{\xi}<-\frac{1}{b} \exp \left(\frac{I}{5 \varepsilon}\right) .
$$

\subsection{Monotonicity: proof of lemma 2.10}

In this section we prove lemma 2.10, Let us first ensure that the following assertions are fulfilled:

1. $\frac{d}{d \varepsilon}(x-y)\left(\bar{C}_{\varepsilon}\right) \rightarrow \infty$ as $\varepsilon \rightarrow 0^{+}$for any choice of $\bar{C}_{\varepsilon}=\bar{A}_{\varepsilon}^{ \pm} ; \bar{E}_{\varepsilon}^{ \pm}$

2. The equation $(y-x)\left(\bar{E}_{\varepsilon}^{-}\right)=2 \pi n$ has root $\varepsilon=\varepsilon_{n}$ for any $n$, and $\varepsilon_{n}=O(1 / n)$.

Consider the cross-section $\Gamma_{0}:=\left\{y=y_{0}\right\}$ for some $y_{0} \in\left[\delta_{+}, \delta_{-}\right]$. Consider the Poincaré map from $\Gamma_{0}$ to $\Gamma=\{y=\pi\}$ in forward and reverse times:

$$
\begin{aligned}
& R_{\varepsilon}^{-}\left(x ; y_{0}\right):=P_{\varepsilon}^{\left\langle-\pi, y_{0}\right]}(x), \\
& R_{\varepsilon}^{+}\left(x ; y_{0}\right):=P_{\varepsilon}^{\left[y_{0},+\pi\right\rangle}(x) .
\end{aligned}
$$

We lift these maps from the circle $S_{x}^{1}$ to the universal cover $\mathbb{R}_{x}^{1}$ continuosly in $\varepsilon$, and denote the result by $\bar{R}_{\varepsilon}^{ \pm}$( $y_{0}$ is considered a fixed parameter).

The proof of the lemma is based on the following proposition:

Proposition 4.7. One can find positive constants $C^{ \pm}$, such that for any fixed $x_{0} \in S^{1}$ and any $\varepsilon>0$ small enough, the following facts hold:

1. $\frac{d \bar{R}_{\varepsilon}^{ \pm}\left(x_{0} ; y_{0}\right)}{d \varepsilon} \rightarrow \mp \infty$ as $\varepsilon \rightarrow 0^{+}$,

2. $\bar{R}_{\varepsilon}^{+}\left(x_{0} ; y_{0}\right)=\frac{C^{+}+O\left(\delta_{+}^{2}\right)+O(\varepsilon)}{\varepsilon}$,

3. $\bar{R}_{\varepsilon}^{-}\left(x_{0} ; y_{0}\right)=\frac{-C^{-}+O\left(\delta_{-}^{2}\right)+O(\varepsilon)}{\varepsilon}$;

This proposition is proved in [GI], (see the proof for points $\bar{d}_{\varepsilon}^{-}$and $\bar{A}_{\varepsilon}^{ \pm}$, pp. 45-46) for some particular system, but the proof can be extended to our case verbatim. 
Proof of Lemma 2.10. Let us recall that

$$
\begin{aligned}
A_{\varepsilon}^{-}:=\left(p_{\varepsilon}^{+}, P_{\varepsilon}\left(p_{\varepsilon}^{+}\right)\right), & A_{\varepsilon}^{+}:=\left(q_{\varepsilon}^{+}, P_{\varepsilon}\left(q_{\varepsilon}^{+}\right)\right), \\
E_{\varepsilon}^{-}:=\left(p_{\varepsilon}^{+}, q_{\varepsilon}^{-}\right), & E_{\varepsilon}^{+}:=\left(q_{\varepsilon}^{+}, p_{\varepsilon}^{-}\right) .
\end{aligned}
$$

By definition, $D_{\varepsilon}^{ \pm}=\left[p_{\varepsilon}^{ \pm}, q_{\varepsilon}^{ \pm}\right]$(see subsection 4.1 and fig. 2.1 on page 4),

$$
\begin{aligned}
& p_{\varepsilon}^{+}=R_{\varepsilon}^{-}\left(\sigma^{+} ; \delta_{+}\right), \quad q_{\varepsilon}^{+}=R_{\varepsilon}^{-}\left(+\pi ; \delta_{+}\right), \\
& p_{\varepsilon}^{-}=R_{\varepsilon}^{+}\left(-\pi ; \delta_{-}\right), \quad q_{\varepsilon}^{-}=R_{\varepsilon}^{+}\left(\sigma^{-} ; \delta_{-}\right) .
\end{aligned}
$$

It is obvious that

$$
P_{\varepsilon}\left(p_{\varepsilon}^{+}\right)=R_{\varepsilon}^{+}\left(\sigma^{+} ; \delta_{+}\right), \quad P_{\varepsilon}\left(q_{\varepsilon}^{+}\right)=R_{\varepsilon}^{+}\left(+\pi ; \delta_{+}\right) .
$$

Therefore,

$$
\begin{aligned}
& (x-y)\left(A_{\varepsilon}^{-}\right)=R_{\varepsilon}^{-}\left(\sigma^{+} ; \delta_{+}\right)-R_{\varepsilon}^{+}\left(\sigma^{+} ; \delta_{+}\right), \\
& (x-y)\left(A_{\varepsilon}^{+}\right)=R_{\varepsilon}^{-}\left(+\pi ; \delta_{+}\right)-R_{\varepsilon}^{+}\left(+\pi ; \delta_{+}\right), \\
& (x-y)\left(E_{\varepsilon}^{-}\right)=R_{\varepsilon}^{-}\left(\sigma^{+} ; \delta_{+}\right)-R_{\varepsilon}^{+}\left(\sigma^{-} ; \delta_{-}\right), \\
& (x-y)\left(E_{\varepsilon}^{+}\right)=R_{\varepsilon}^{-}\left(+\pi ; \delta_{+}\right)-R_{\varepsilon}^{+}\left(-\pi ; \delta_{+}\right) .
\end{aligned}
$$

Using (4.45), it is easy to show that assertion 1 of the lemma follows from assertion 1 of proposition 4.7, and assertion 2 of the lemma follows from the assertions 2 and 3 of the same proposition.

\section{Influence of the jump point: proof of tech- nical propositions}

\subsection{Dynamics near jump point}

\subsubsection{Composition of the Poincaré maps}

In this section we will prove Theorem 4.3. Let us show that for some $\lambda>0$, derivative of the Poincaré map $R_{\varepsilon}=Q_{\varepsilon}^{-1}=P_{\varepsilon}^{\left\langle-\pi, \alpha^{+}\right]}$can be written in the following form:

$$
\ln R_{\varepsilon}^{\prime}(x)=\frac{C\left(\delta_{+}\right)+O\left(\varepsilon^{\lambda}\right)}{\varepsilon}
$$

where $C\left(\delta_{+}\right)<0$ is continuous and tends to 0 monotonically as $\delta_{+} \rightarrow 0^{+}$.

For some $\varepsilon>0$, consider the two cross-sections

$$
\Gamma_{\varepsilon}^{1}=\left\{(x, y) \mid y=\tau^{+}-\varepsilon^{\nu}\right\}, \Gamma_{\varepsilon}^{2}=\left\{(x, y) \mid y=\tau^{+}+\varepsilon^{\mu}\right\},
$$


where $\mu, \nu \in(0,1)$ are constants to be defined later.

Consider $R_{\varepsilon}$ as a composition:

$$
\begin{gathered}
R_{\varepsilon}: \Gamma^{+} \stackrel{R_{\subsetneq}^{1}}{\rightarrow} \Gamma_{\varepsilon}^{2} \stackrel{R_{\subsetneq}^{2}}{\rightarrow} \Gamma_{\varepsilon}^{1} \stackrel{R_{\xi}^{3}}{\rightarrow} \Gamma \\
R_{\varepsilon}=R_{\varepsilon}^{3} \circ R_{\varepsilon}^{2} \circ R_{\varepsilon}^{1}
\end{gathered}
$$

Take the logarithmic derivative of (5.4):

$$
\ln R_{\varepsilon}^{\prime}=\ln \left(R_{\varepsilon}^{3}\right)^{\prime} \circ R_{\varepsilon}^{2} \circ R_{\varepsilon}^{1}+\ln \left(R_{\varepsilon}^{2}\right)^{\prime} \circ R_{\varepsilon}^{1}+\ln \left(R_{\varepsilon}^{1}\right)^{\prime} .
$$

Lemma 3.5 implies that the second term of the sum is $O\left(\varepsilon^{\min (\mu, \nu)-1}\right)$. We will prove that

$$
\begin{gathered}
\left|\ln \left(R_{\varepsilon}^{3}\right)^{\prime}\right|<C \varepsilon^{\nu-1}, \\
\ln \left(R_{\varepsilon}^{1}\right)^{\prime}=\frac{C\left(\delta_{+}\right)+O\left(\varepsilon^{\mu}\right)}{\varepsilon}, \quad C\left(\delta_{+}\right)<0 .
\end{gathered}
$$

Taking $\lambda=\min (\mu, \nu)$ and using these estimates in (5.5), we obtain the the desired estimate and thus complete the proof of theorem 4.3 ,

\subsubsection{Dynamics far from the true slow curve}

The following proposition generalizes theorem 3.1. It allows us to estimate the derivative of the Poincaré map from some given cross-section to the the cross-section which slowly approaches the jump point.

Let us move the origin to $G^{+}=\left(\sigma^{+}, \tau^{+}\right)$. Then cross-section $\Gamma=\{y=$ $-\pi\}$ becomes $\{y=a\}$ for some $a>0$ (which can be chosen arbitrary by an appropriate coordinate change).

Define the map:

$$
Q_{\varepsilon}^{3}=\left(R_{\varepsilon}^{3}\right)^{-1}=P_{\varepsilon}^{\left[a,-\varepsilon^{\nu}\right\rangle}: \Gamma \rightarrow \Gamma_{\varepsilon}^{1} .
$$

Proposition 5.1. The derivative of $Q_{\varepsilon}^{3}$ can be estimated as follows:

$$
\left|\ln \left(Q_{\varepsilon}^{3}\right)^{\prime}\right|=\left|\ln \left(R_{\varepsilon}^{3}\right)^{\prime}\right|<C \varepsilon^{\nu-1} .
$$

Proposition 5.1 is proved in subsection 5.2 . 


\subsubsection{True slow curve near the jump point}

Proposition 5.2. Assume that the conditions of Proposition 5.1 are satisfied for any $u \in \Gamma^{+}$in some neighborhood of the slow curve $M^{+}$. Then, the following equality holds:

$$
\ln \left(R_{\varepsilon}^{1}\right)^{\prime}(u)=\frac{C\left(\delta_{+}\right)+O\left(\varepsilon^{\mu}\right)}{\varepsilon},
$$

where $C\left(\delta_{+}\right)<0$ is continuous and tends to 0 monotonically as $\delta_{+} \rightarrow 0$.

To prove this proposition we need a description of the asymptotics of the true slow curve near the jump point:

Theorem 5.3 ([MR], p. 119). There exists a $\mu \in(0,1 / 3)$ such that the function defining true slow curve, $x=s(y, \varepsilon)$, admits the following representation for $y \in\left[\varepsilon^{\mu}, \delta_{+}\right]$:

$$
s(y, \varepsilon)=s(y)+O\left(\varepsilon^{2 / 3-\mu / 2}\right),
$$

where $s(y)$ defines the slow curve.

This theorem can be deduced by applying a trivial coordinate change to representation (16.10) from the cited work.

Remark 5.4. In the definition of the cross-section $\Gamma_{\varepsilon}^{2}$ we set $\mu$ equal to the value given by theorem 5.3 .

Proof of the Proposition 5.2. Fix a cross-section $\Gamma^{\prime}=\left\{y=\delta^{\prime}\right\}$ for $\delta^{\prime} \ll \delta_{+}$. Assume that $\varepsilon$ is small enough to assure $\delta^{\prime}>\varepsilon^{\mu}$. We represent the map $R_{\varepsilon}^{1}$ as a composition:

$$
R_{\varepsilon}^{1}: \Gamma^{+} \stackrel{H_{\varepsilon}^{1}}{\rightarrow} \Gamma^{\prime} \stackrel{H_{\varepsilon}^{2}}{\rightarrow} \Gamma_{\varepsilon}^{2} .
$$

Suppose that the trajectory passing through the point $\left(u, \delta_{+}\right)$is the graph of a function $x=x(y, \varepsilon)$. Theorem 3.2 implies that $H_{\varepsilon}^{1}$ is a linear contraction with an exponential rate of order $O\left(e^{-C / \varepsilon}\right)$ in normalizing charts. Fix a segment of $\Gamma^{+}$that intersects the slow curve $M^{+}$. Due to the exponential contraction, any trajectory that crosses this segment is quickly attracted to true slow curve in reverse time. We have:

$$
\left|x\left(\delta^{\prime}, \varepsilon\right)-s^{+}\left(\delta^{\prime}, \varepsilon\right)\right| \leq \text { const } e^{-C / \varepsilon}
$$

With an appropriate choice of $\delta^{\prime}$, we can move $\Gamma^{\prime}$ arbitrarily close to $\Gamma_{\varepsilon}^{2}$ for $\varepsilon$ small enough. Thefore, the trajectory spends much more time during the 
transition from $\Gamma^{+}$to $\Gamma^{\prime}$ than during the transition from $\Gamma^{\prime}$ to $\Gamma_{\varepsilon}^{2}$. Hence, lemma 3.5 implies that for any $y \in\left[\varepsilon^{\mu}, \delta^{\prime}\right]$ the following estimate holds:

$$
\left|x(y, \varepsilon)-s^{+}(y, \varepsilon)\right| \leq \text { const } e^{-C / \varepsilon}
$$

In other words, the corresponding trajectory on the segment from $\Gamma^{\prime}$ to $\Gamma_{\varepsilon}^{2}$ moves exponentially close to the true slow curve.

Variational equations imply:

$$
\begin{aligned}
& \ln \left(R_{\varepsilon}^{1}\right)^{\prime}(u)=\ln \left(H_{\varepsilon}^{1}\right)^{\prime}(u)+\ln \left(H_{\varepsilon}^{2}\right)^{\prime} \circ H_{\varepsilon}^{1}(u)= \\
& =\frac{-c_{3}\left(\delta^{\prime}, \delta_{+}\right)}{\varepsilon}+\frac{1}{\varepsilon} \int_{\delta^{\prime}}^{\varepsilon^{\mu}} f^{\prime}(x(y, \varepsilon), y, \varepsilon) d y+O(1),
\end{aligned}
$$

where $c_{3}\left(\delta^{\prime}, \delta_{+}\right)>0$.

Due to (15.14), one can replace $x(y, \varepsilon)$ by $s^{+}(y, \varepsilon)$ in the second term of (5.15). Moreover, using representation (5.11), we obtain:

$$
\begin{gathered}
\ln \left(H_{\varepsilon}^{2}\right)^{\prime}=\frac{1}{\varepsilon} \int_{\delta^{\prime}}^{\varepsilon^{\mu}} f_{x}^{\prime}(x(y, \varepsilon), y, \varepsilon) d y=\frac{1}{\varepsilon} \int_{\delta^{\prime}}^{\varepsilon^{\mu}} f_{x}^{\prime}\left(s^{+}(y, \varepsilon), y, \varepsilon\right) d y+O(1)= \\
=\frac{1}{\varepsilon}\left[\int_{\delta^{\prime}}^{\varepsilon^{\mu}} f_{x}^{\prime}\left(s^{+}(y), y, 0\right) d y+O\left(\varepsilon^{2 / 3-\mu / 2}\right)\right]=\frac{1}{\varepsilon}\left[\int_{\delta^{\prime}}^{0} f_{x}^{\prime}\left(s^{+}(y), y, 0\right) d y+O\left(\varepsilon^{\mu}\right)\right]= \\
=\frac{-c_{4}\left(\delta^{\prime}\right)+O\left(\varepsilon^{\mu}\right)}{\varepsilon}
\end{gathered}
$$

Denoting $C=-\left(c_{3}+c_{4}\right)$ and substituting (5.16) into (5.15) , we obtain (5.10). The number $C$ is $\delta_{+}$-monotonic, because expressions under the integral sign in (5.15) and (5.16) are positive.

\subsection{Distortion lemma: proof of proposition 5.1}

In this section we prove proposition 5.1. Instead of considering the Poincaré map from one vertical cross-section to another, we consider Poincaré map from the horizontal cross-section $x=0$ to itself. We will iterate this Poincaré map and estimate the derivative of these iterations using the Distortion Lemma due to Denjoy and Schwartz. To apply this lemma we need some additional estimates obtained from the variational equations.

\subsubsection{Vertical Poincaré map}

Proof of the proposition 5.1. Along with the system (2.1) we will consider two auxilliary systems with the same phase portraits for any given $\varepsilon$ : 


$$
\dot{x}=v(x, y, \varepsilon), \quad \dot{y}=\varepsilon ;
$$

and

$$
\dot{x}=1, \quad \dot{y}=\varepsilon w(x, y, \varepsilon)
$$

where

$$
v(x, y, \varepsilon)=\frac{f(x, y, \varepsilon)}{g(x, y, \varepsilon)}, \quad w(x, y, \varepsilon)=\frac{g(x, y, \varepsilon)}{f(x, y, \varepsilon)} .
$$

According to remark 2.7, without loss of generality we can assume that $g=1$ and then $v=f, w=1 / f$.

Nondegenerecity conditions (2.5) imply that for $a<y<0$ and for some positive constants $c_{v}, C_{v}, c_{w}, C_{w}$ the following equivalent estimates hold:

$0<-c_{v}(y+O(\varepsilon))<v(x, y, \varepsilon)<C_{v} \quad \Leftrightarrow \quad 0<c_{w}<w(x, y, \varepsilon)<-\frac{C_{w}}{y+O(\varepsilon)}$

For the sake of simplicity, we will rescale the coordinate $x \mapsto 2 \pi x$ so that $x$ becomes a coordinate modulo $\mathbb{Z}$.

Denote the line $\{x=0\}=\{x=1\}$ by $\Sigma$, and the Poincaré map $\Sigma \rightarrow \Sigma$ by $\psi$ (see fig. 5.4). Recall that we are considering the following cross-sections:

$$
\begin{gathered}
\Gamma=\{(x, y) \mid y=a\} \\
\Gamma_{\varepsilon}^{1}=\left\{(x, y) \mid y=-\varepsilon^{\nu}\right\} .
\end{gathered}
$$

For brevity, we will write $\Gamma_{\varepsilon}$ instead of $\Gamma_{\varepsilon}^{1}$.

Let us define a correspondence map $\gamma$ from the cross-section $\Gamma$ to the semi-interval $J_{0} \subset \Sigma$ in forward time (see fig. 5.4). Note that $\gamma$ is not countinous at 0 , so in the following analysis we will replace the circle $\Gamma$ by the semi-interval $[0,1)$, where $\gamma$ is continious. Consequently, let us denote:

$$
J_{k+1}:=\psi\left(J_{k}\right),\left.\quad \psi\right|_{J_{k}}=: \psi_{k}, \quad k \geq 1
$$

Obviously, the semi-intervals $J_{k}$ do not intersect each other and the right end of the $k$-th interval coincides with the left end of $(k+1)$-th interval. Define $N=N(\varepsilon)$ in such way that $J_{N}$ intersects $\Gamma_{\varepsilon}$. Denote by $\tau$ the intersection point of $\Gamma_{\varepsilon}$ and the trajectory passing thought $(0, a)$.

Let us define $\gamma_{\varepsilon}: \Gamma_{\varepsilon} \rightarrow J_{N}$ in the following way. On the interval $[0, \tau\rangle$ (resp., $[\tau, 1\rangle$ ) it coincides with the correspondence map for the phase flow of (2.1) in the reverse (resp., forward) time. Defined this way, $\gamma_{\varepsilon}$ will be 


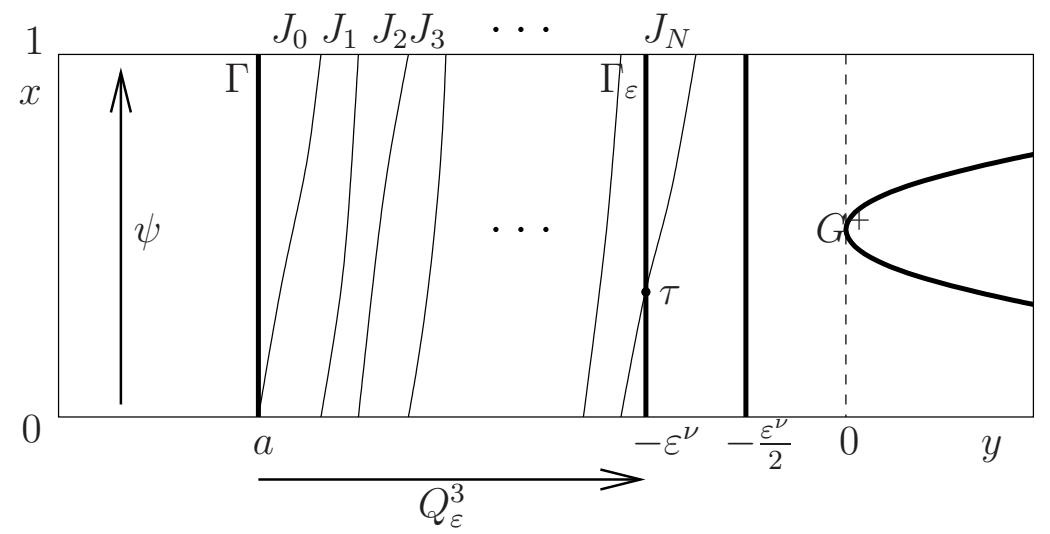

Figure 5.4: Correspondence maps

discontinious in $\tau$, but its inverse $\gamma_{\varepsilon}^{-1}$ continiuosly projects $J_{N}$ to the circle $\Gamma_{\varepsilon}$ along the phase curves.

Now $Q_{\varepsilon}^{3}$ may be represented by the following composition:

$$
\begin{gathered}
Q_{\varepsilon}^{3}: \Gamma \stackrel{\gamma}{\rightarrow} J_{0} \stackrel{\psi_{0}}{\rightarrow} \ldots \stackrel{\psi_{N-1}}{\rightarrow} J_{N} \stackrel{\gamma_{\varepsilon}^{-1}}{\rightarrow} \Gamma_{\varepsilon} \\
Q_{\varepsilon}^{3}=\gamma_{\varepsilon}^{-1} \circ \psi_{N-1} \circ \cdots \circ \psi_{0} \circ \gamma=\gamma_{\varepsilon}^{-1} \circ F_{N-1} \circ \gamma
\end{gathered}
$$

The chain rule implies:

$$
\left|\ln Q_{\varepsilon}^{\prime}\right| \leq\left|\max _{J_{N}} \ln \right|\left(\gamma_{\varepsilon}^{-1}\right)^{\prime}||+\left|\max _{J_{0}} \ln F_{N-1}^{\prime}\right|+\left|\max _{\Gamma} \ln \right| \gamma^{\prime}||
$$

We will obtain the following estimates for the terms of this sum.

$$
\begin{aligned}
|\ln | \gamma_{\varepsilon}^{\prime}||<C \varepsilon^{\nu-1} \\
\left|\ln F_{N-1}^{\prime}\right| \leq(\nu-1) \ln \varepsilon+\mathrm{const}+o(1) \\
|\ln | \gamma^{\prime}|| \leq \ln \frac{1}{\varepsilon}
\end{aligned}
$$

These estimates are obtained below and they justify (5.9).

Remark 5.5. Inequality (5.21) can be applied to every point of $\Gamma$ excluding 0 , because $\gamma$ and $\gamma_{\varepsilon}$ are discontinuous in 0 and $\tau$ respectively. However, we can still obtain necessary inequalities on the whole $\Gamma$. To this end, we have to define another cross-section $\Sigma$ (e.g. $\Sigma=\left\{x=\frac{1}{2}\right\}$ ) and use the same arguments for this new cross-section to obtain the inequality for $x=0$. 


\subsubsection{Application of the Distortion Lemma}

In this section we will obtain (5.23). First, we give the statement of the Distortion Lemma.

Defenition 5.6. For any diffeomorphism $\psi: A \rightarrow B$ define its distortion rate as:

$$
\kappa(\psi)=\ln \frac{\max _{A} \psi^{\prime}}{\min _{A} \psi^{\prime}}=\max _{x, y \in A} \ln \frac{\psi^{\prime}(x)}{\psi^{\prime}(y)} .
$$

Lemma 5.7 (Distortion Lemma ([De], [Sch $])$ ). Consider a sequence of arbitrary intervals and their orientation-preserving diffeomorphisms:

$$
J_{0} \stackrel{\psi_{0}}{\rightarrow} J_{1} \stackrel{\psi_{1}}{\rightarrow} \ldots \stackrel{\psi_{N-1}}{\rightarrow} J_{N} .
$$

Then the following estimate holds for the composition $F_{N-1}:=\psi_{N-1} \circ \cdots \circ \psi_{0}$ of these maps:

$$
\kappa\left(F_{N-1}^{\prime}\right) \leq \max _{i} \max _{x \in J_{i}} \cdot\left|\frac{\psi_{i}^{\prime \prime}(x)}{\psi_{i}^{\prime}(x)}\right| \sum_{k=0}^{N-1}\left|J_{k}\right| .
$$

This lemma easily follows from the subadditivity (under the composition) of the distortion rate and the mean value theorem, which implies that:

$$
\kappa(\psi)=\max _{x, y \in A}\left(\ln \psi^{\prime}(x)-\ln \psi^{\prime}(y)\right) \leq \max _{z \in A}\left|\frac{\psi^{\prime \prime}(z)}{\psi^{\prime}(z)}\right| \cdot|A| .
$$

Our strategy is to apply the Distortion Lemma to composition $F_{N-1}$ of the iterations of the vertical Poincaré map $\psi$ (see subsection 5.2.1). To this end, we have to estimate the sum of lengths for $\left\{J_{k}\right\}_{k=0}^{N-1}$ and the distortion rate of $\psi$. In the rest of this subsection we consider system (5.18).

By construction, the intervals $J_{k}$ do not intersect each other. Therefore, to estimate the sum of their lengths it is sufficient to control the last interval $J_{N}:=\left[x_{N}, x_{N+1}\right]$.

Proposition 5.8. For any $\nu<1 / 2$ and $\varepsilon$ small enough, we have:

$$
\begin{gathered}
\left|J_{N}\right| \leq \varepsilon^{\nu} \\
x_{N+1}<-\frac{1}{2} \varepsilon^{\nu}
\end{gathered}
$$

Proof. Let us denote the projections of the phase space to $\Sigma$ along the phase curves in forward $(+)$ and reverse $(-)$ time as $T^{ \pm}$. Obviosly,

$$
\begin{aligned}
J_{N}=\left[T^{-}\left(\tau,-\varepsilon^{\nu}\right)\right. & \left., T^{+}\left(\tau,-\varepsilon^{\nu}\right)\right) \subset \\
& \subset\left[T^{-}\left(1,-\varepsilon^{\nu}\right), T^{+}\left(0,-\varepsilon^{\nu}\right)\right]=\left[\psi^{-1}\left(-\varepsilon^{\nu}\right), \psi\left(-\varepsilon^{\nu}\right)\right] .
\end{aligned}
$$


Let us consider the trajectory that passes through the point $\left(0,-\varepsilon^{\nu}\right)$. The second equation of the system (5.18) is:

$$
\dot{y}=\varepsilon w(x, y, \varepsilon)
$$

By (5.20), the right-hand side of this equation is positive and can be estimated from above by $-\varepsilon \frac{C_{w}}{y+O(\varepsilon)}$ (recall that in the domain under considertion $y<0$ ). Solving the equation

$$
\dot{y}_{*}=-\varepsilon \frac{C_{w}}{y_{*}+O(\varepsilon)},
$$

we find that

$$
y_{*}\left(t ; y_{0}\right)=-\sqrt{y_{0}^{2}-2 \varepsilon C_{w} t+O(\varepsilon)}, \quad y_{*}\left(0 ; y_{0}\right)=y_{0}<0 .
$$

Therefore,

$$
\begin{aligned}
T^{+}\left(0,-\varepsilon^{\nu}\right)=y\left(1 ;-\varepsilon^{\nu}\right) \leq y_{*}\left(1 ;-\varepsilon^{\nu}\right) & =-\sqrt{\varepsilon^{2 \nu}+O(\varepsilon)}= \\
& =-\varepsilon^{\nu} \sqrt{1+O\left(\varepsilon^{1-2 \nu}\right)}<-\frac{1}{2} \varepsilon^{\nu}
\end{aligned}
$$

The last inequality holds for $\varepsilon$ small enough if $\nu<1 / 2$.

Similar arguments show that $T^{-}\left(0,-\varepsilon^{\nu}\right)>-\frac{3}{2} \varepsilon^{\nu}$, which implies the proposition.

Remark 5.9. Obviosly, (5.20) implies the following estimates for some positive constants $C_{J_{N}}, C_{J_{0}}$ and $c_{J_{0}}$ :

$$
\left|J_{N}\right| \geq C_{J_{N}} \varepsilon, \quad c_{J_{0}} \varepsilon \leq\left|J_{0}\right| \leq C_{J_{0}} \varepsilon
$$

Proposition 5.10. For any $\nu<1 / 2$ the derivative of the Poincaré map $\psi$ : $\Sigma \rightarrow \Sigma$ admits the following estimate in the domain $a<y<-\varepsilon^{\nu}$ :

$$
\begin{gathered}
\psi^{\prime} \geq \frac{1}{2}, \\
\psi^{\prime \prime} \leq C \varepsilon^{1-4 \nu} .
\end{gathered}
$$

Proof. Proposition 5.8 implies that a trajectory starting from the domain $\left\{a \leq y \leq-\varepsilon^{\nu}\right\}$ does not leave the domain $\left\{a-O(\varepsilon) \leq y \leq-\varepsilon^{\nu} / 2\right\}$ until it intersects $\Sigma$.

Let $y=y\left(t ; y_{0}\right)$ be the $y$-coordinate of the solution of the system (5.18) with the initial conditions $x(0)=0, y(0)=y_{0}$. Obviously, 


$$
\psi\left(y_{0}\right)=y\left(1 ; y_{0}\right)
$$

Taking the derivative with respect to $y_{0}$, we obtain:

$$
\psi^{\prime}\left(y_{0}\right)=\frac{\partial y\left(1 ; y_{0}\right)}{\partial y_{0}}=: y_{y_{0}}^{\prime}\left(1 ; y_{0}\right) .
$$

The variational equation for $y_{y_{0}}^{\prime}$ has the form:

$$
\dot{y}_{y_{0}}^{\prime}=\varepsilon \frac{\partial w}{\partial y} y_{y_{0}}^{\prime}, \quad y_{y_{0}}^{\prime}\left(0 ; y_{0}\right)=1
$$

Therefore,

$$
y_{y_{0}}^{\prime}\left(t ; y_{0}\right)=\exp \int_{0}^{t} \varepsilon \frac{\partial w}{\partial y}\left(x\left(\xi ; y_{0}\right), y\left(\xi ; y_{0}\right), \varepsilon\right) d \xi \text {. }
$$

For $a<y<-\frac{1}{2} \varepsilon^{\nu}$ the derivative $\frac{\partial w}{\partial y}$ can be estimated as follows:

$$
\left|\frac{\partial w}{\partial y}\right|=\left|\frac{\partial}{\partial y} \frac{g(x, y, \varepsilon)}{f(x, y, \varepsilon)}\right|=\left|\frac{g_{y}^{\prime} f-f_{y}^{\prime} g}{f^{2}}\right| \leq \frac{C}{(y+O(\varepsilon))^{2}} \leq \frac{C_{1}}{\varepsilon^{2 \nu}} .
$$

Therefore, for $\nu<\frac{1}{2}$ and $\varepsilon$ small enough,

$$
\psi^{\prime}\left(y_{0}\right) \geq \exp \left(-\varepsilon \frac{C_{1}}{\varepsilon^{2 \nu}}\right)=\exp \left(-C_{1} \varepsilon^{1-2 \nu}\right)>\frac{1}{2},
$$

which proves (5.36).

To obtain (5.37) we take the derivative of (5.41) with respect to $y_{0}$ for $t=$ 1:

$$
\begin{array}{r}
\psi^{\prime \prime}\left(y_{0}\right)= \\
\frac{\partial}{\partial y_{0}} y_{y_{0}}^{\prime}\left(1 ; y_{0}\right)=\frac{\partial}{\partial y_{0}} \exp \int_{0}^{1} \varepsilon \frac{\partial w}{\partial y}\left(x\left(\xi ; y_{0}\right), y\left(\xi ; y_{0}\right), \varepsilon\right) d \xi= \\
=\varepsilon \psi^{\prime}\left(y_{0}\right) \int_{0}^{1} \frac{\partial^{2} w}{\partial y^{2}} y_{y_{0}}^{\prime}\left(\xi ; y_{0}\right) d \xi \leq \varepsilon\left(\max _{\xi \in[0,1]} y_{y_{0}}^{\prime}\left(\xi ; y_{0}\right)\right)^{2} \frac{C_{2}}{\varepsilon^{4 \nu}} \leq \\
\leq C \varepsilon^{1-4 \nu} \exp \left(C \varepsilon^{1-2 \nu}\right) \leq C \varepsilon^{1-4 \nu}
\end{array}
$$

where $\frac{\partial^{2} w}{\partial y^{2}}$ was estimated from above by $\frac{C_{2}}{\varepsilon^{4 \nu}}$ (the justification is similar to (5.42) $)$, and $y_{y_{0}}^{\prime}$ was estimated using (5.40) and (5.42).

Corrollary 5.11. For $\nu<1 / 4$ and $\varepsilon \rightarrow 0$ we have:

$$
\frac{\psi^{\prime \prime}}{\psi^{\prime}} \leq C \varepsilon^{1-4 \nu} \rightarrow 0
$$


Therefore, the Distortion Lemma gives the following estimate for $F_{N-1}$ (for brevity, we omit the index $N-1$ below):

$$
\ln \frac{\max _{J_{0}} F^{\prime}}{\min _{J_{0}} F^{\prime}} \leq C \max _{\left[a,-\varepsilon^{\nu}\right]} \frac{\psi^{\prime \prime}}{\psi^{\prime}}=o(1) .
$$

It implies that

$$
\ln \max _{J_{0}} F^{\prime}=\ln \min _{J_{0}} F^{\prime}+o(1)
$$

At the same time, the mean value theorem implies that for any $\varepsilon>0$ small enough there exists some point $y_{0} \in J_{0}$, such that

$$
F^{\prime}\left(y_{0}\right)=\frac{\left|J_{N}\right|}{\left|J_{0}\right|} .
$$

According to the estimates (5.28) and (5.35), we have:

$$
\begin{aligned}
\min F^{\prime} & \leq F^{\prime}\left(y_{0}\right) \leq C_{1} \varepsilon^{\nu-1} \\
\max F^{\prime} & \geq F^{\prime}\left(y_{0}\right) \geq C_{2} .
\end{aligned}
$$

Taking the lograhithm of (5.48) and using (5.47), we have:

$$
\begin{aligned}
\ln \max F^{\prime} & \leq \ln C_{1} \varepsilon^{\nu-1}+o(1), \\
\ln \min F^{\prime} & \geq \text { const }+o(1) .
\end{aligned}
$$

Therefore, (5.23) is justified.

\subsubsection{The projection to the horizontal cross-section}

In this subsection inequalities (5.22) and (5.24) are proved. Consider the system (5.17).

Proposition 5.12. For the map $\gamma: \Gamma \rightarrow J_{0}$ the following estimate holds:

$$
|\ln | \gamma^{\prime}|| \leq \ln \frac{1}{\varepsilon}+O(1) \text {. }
$$

Proof. Let us fix $x_{0}$ and define $y_{0}:=\gamma\left(x_{0}\right)$. We present $\gamma(x)$ as a composition (see fig. [5.5):

$$
\gamma: \Gamma \stackrel{R}{\rightarrow} \Gamma^{\prime} \stackrel{\tilde{\gamma}}{\rightarrow} \Sigma,
$$

where $\Gamma^{\prime}=\left\{y=y_{0}\right\}$ is the shifted vertical cross-section, $R$ is the correspondence map from $\Gamma$ to $\Gamma^{\prime}$, and $\tilde{\gamma}$ is the correspondence map from some 


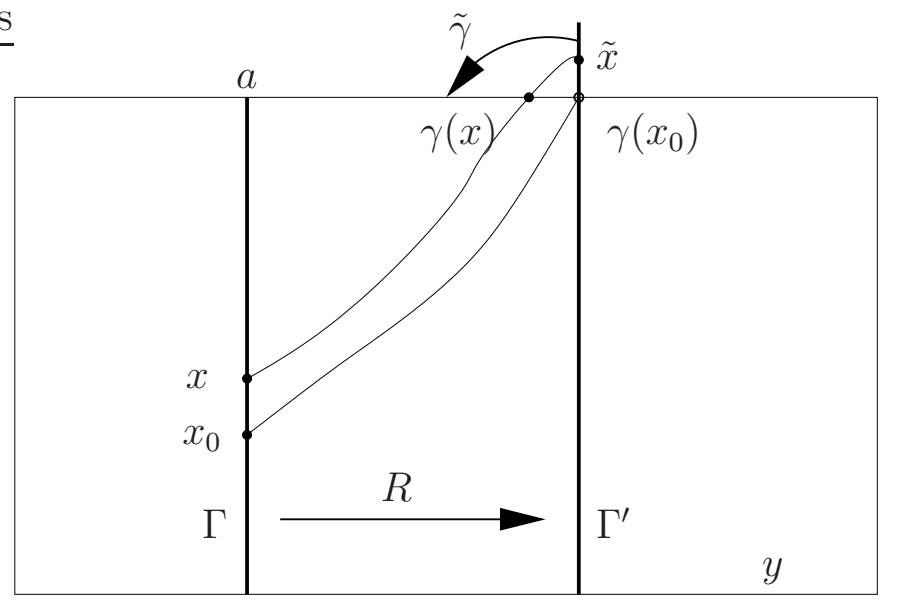

Figure 5.5: Estimate of the derivative $\gamma$

neighborhood of the point $x=0$ on $\Gamma^{\prime}$ to some neighborhood of $y=y_{0}$ on $\Sigma$, which is defined in the following way:

$$
\tilde{\gamma}( \pm \tilde{x})=T^{\mp}\left(\tilde{x}, y_{0}\right)
$$

Recall that $T^{ \pm}$are the projections of the phase space to $\Sigma$ along the trajectories of the system in forward $(+)$ and reverse $(-)$ time.

It is easy to see that $\tilde{\gamma}^{\prime}(1)=-\varepsilon w\left(0, \gamma\left(x_{0}\right), \varepsilon\right)$, because in a small neighborhood of $\left(1, y_{0}\right)$ the map $\tilde{\gamma}$ is close to the linear projection from $\Gamma^{\prime}$ to $\Sigma$ along the vector $\left(1,-\varepsilon w\left(1, y_{0}, \varepsilon\right)\right)$.

The function $w$ is bounded away from zero and infinity in some neighborhood of the cross-section $\Gamma$. Therefore $\left|\tilde{\gamma}^{\prime}\right|$ is of order $\varepsilon$.

Note, that the distance between $\Gamma$ and $\Gamma^{\prime}$ is of order $\varepsilon$, and therefore the time of the transition between these cross-sections along the phase curves of the system (5.17) is bounded from above. Hence, the derivative of $R$ is bounded from above (what follows from the variational equations). Therefore, estimates for $\tilde{\gamma}^{\prime}$ and $\gamma^{\prime}$ coincide.

Proposition 5.13. For every point $x_{0} \neq \tau$, the following estimate holds:

$$
|\ln | \gamma_{\varepsilon}^{\prime}\left(x_{0}\right)||<C \varepsilon^{\nu-1}
$$

Proof. Like in the previous proposition, we fix $x_{0}$ and represent $\gamma_{\varepsilon}$ as a composition:

$$
\gamma_{\varepsilon}: \Gamma_{\varepsilon} \stackrel{R_{\varepsilon}}{\rightarrow} \Gamma_{\varepsilon}^{\prime} \stackrel{\tilde{\gamma}_{\varepsilon}}{\rightarrow} J_{N}
$$


where $\Gamma_{\varepsilon}^{\prime}=\left\{y=\gamma_{\varepsilon}\left(x_{0}\right)\right\}$.

According to proposition 5.8, all the trajectories starting at $\Gamma_{\varepsilon}$ stay in the strip $\left\{-\frac{3}{2} \varepsilon^{\nu}<y<-\frac{1}{2} \varepsilon^{\nu}\right\}$ until they cross $\Sigma$ in forward or reverse time. Therefore, the trajectory of (5.17) spends time $t_{*}=C_{1} \varepsilon^{\nu-1}$ before it intersects $\Sigma$. Due to the smoothness of $v(x, y, \varepsilon)$ on the whole torus, the variational equations imply that

$$
\left|\ln R^{\prime}\right| \leq C_{2} t_{*} \leq C \varepsilon^{\nu-1} .
$$

Applying arguments from the previous proposition to $\tilde{\gamma}_{\varepsilon}^{\prime}$, we obtain:

$$
\tilde{\gamma}_{\varepsilon}^{\prime}(0)=-\varepsilon w\left(1, y_{0}, \varepsilon\right), \quad\left|\ln \tilde{\gamma}_{\varepsilon}^{\prime}(0)\right|<(\nu-1) \ln \varepsilon+O(1) \ll \varepsilon^{\nu-1} .
$$

Provided (5.55) and (5.56), the chain rule justifies (5.53).

Remark 5.14. We can put arbitrary $\nu$ from the interval $(0,1 / 4)$ into the definition of the cross-section $\Gamma_{\varepsilon}=\Gamma_{\varepsilon}^{1}$. 


\section{References}

[A1] O. Anosova, On Invariant Manifolds in Singulalry Perturbed Systems, J. Dyn. Control. Sys., 1999, vol. 5, no 4, pp. 501-507.

[A2] O. Anosova, Invariant Manifolds in Singularly Perturbed Systems, Proceedings of the Steklov Institue of Mathematics, 2002, vol. 236,pp. 19-24.

[De] A. Denjoy. Sur les courbes définies par des équations différentielles à la surface du tore. J. Math. Pure et Appl, 11, (1932), pp. 333-375.

[Di] M. Diener, The canard unchained or how fast/slow dynamical systems bifurcate, The Mathematical Intelligencer 6 (1984), pp. 38-48.

[DR] F. Dumortier and R. Roussarie, Canard cycles and center manifolds, Mem. Amer. Math. Soc., vol. 121, no 577, 1996.

[F] N. Fenichel, Geometric singular perturbation theory for ordinary differential equations, J. of Diff. Eq., 31 (1979), pp. 53-98.

[GI] J. Guckenheimer, Yu. S. Ilyashenko, The Duck and the Devil: Canards on the Staircase, Moscow Math. J., Volume 1, Number 1, 2001, pp. $27-$ 47 .

[KS] M. Krupa, P. Szmolyan, Extending geometric singular perturbation theory to nonhyperbolic points — fold and canard points in two dimensions, SIAM J. Math. Anal., Vol 33, No 2, pp. 286-314.

[MR] E.F. Mishchenko, N.Kh. Rozov, Differential equations with small parameters and relaxation oscillations, Plenum Press, 1980.

[Sch] A. Schwartz. A generalization of Poincaré-Bendixon theorem to closed two dimensional manifolds. Amer. J. Math., 85 (1963), pp. 453-458. 